\title{
ON A SINGULAR LIOUVILLE-TYPE EQUATION AND THE ALEXANDROV ISOPERIMETRIC INEQUALITY.
}

\author{
DANIELE BARTOLUCCI ${ }^{(1, \dagger)}$, DANIELE CASTORINA ${ }^{(2, \ddagger)}$
}

\begin{abstract}
We obtain a generalized version of an inequality, first derived by C. Bandle in the analytic setting, for weak subsolutions of a singular Liouville-type equation. As an application we obtain a new proof of the Alexandrov isoperimetric inequality on singular abstract surfaces. Interestingly enough, motivated by this geometric problem, we obtain a seemingly new characterization of local metrics on Alexandrov's surfaces of bounded curvature. At least to our knowledge, the characterization of the equality case in the isoperimetric inequality in such a weak framework is new as well.
\end{abstract}

Keywords: Singular Liouville-type equations, Alexandrov's Isoperimetric inequality, Surfaces of Bounded Curvature.

\section{INTRODUCTION}

Let $\Omega \subset \mathbb{R}^{2}$ be an open, smooth and bounded domain, $K$ a measurable function on $\Omega, \omega$ be a signed measure of bounded total variation in $\Omega$ and $\omega=\omega_{+}-\omega_{-}$be its Jordan decomposition, that is, for a Borel set $E \subseteq \Omega, \omega_{ \pm}(E)=\sup _{U \subset E}( \pm \omega(U))$. Then $\omega_{ \pm}$are non negative and mutually orthogonal measures of bounded total variation on $\Omega$ and we define $f=f_{+}-f_{-}$, where $f_{+}$and $f_{-}$are two superharmonic functions constructed as follows,

$$
f_{ \pm}(x)=h_{ \pm}(x)+\int_{\Omega} G(x, y) d \omega_{ \pm}(y)
$$

where $h_{ \pm}$are harmonic in $\Omega$. Here $G(x, y)$ denotes the Green's function of $-\Delta$ in $\Omega$. We are concerned with some quantitative estimates for subsolutions of the Liouville-type equation,

$$
-\Delta u=2 K e^{f} e^{u} \text { in } \Omega .
$$

2010 Mathematics Subject classification: 35B45, 35J75, 35R05, 35R45, 30F45, 53B20.

${ }^{(1)}$ Daniele Bartolucci, Department of Mathematics, University of Rome "Tor Vergata",

Via della ricerca scientifica n.1, 00133 Roma, Italy. e-mail:bartoluc@mat.uniroma2.it.

${ }^{(2)}$ Daniele Castorina, Dipartimento di Matematica, Università di Padova,

Via Trieste 63, 35121 Padova, Italy. e-mail: castorin@math.unipd.it.

${ }^{(\dagger)}$ Research partially supported by FIRB project Analysis and Beyond and by PRIN project 2012, ERC PE1_11,

"Variational and perturbative aspects in nonlinear differential problems".

${ }^{(\ddagger)}$ Research partially supported by project Bando Giovani Studiosi 2013 - Università di Padova - GRIC131695. 
D.B. \& D.C.

By assuming $\Omega$ simply connected, $\partial \Omega$ analytic, $u$ an analytic and $C^{0}(\bar{\Omega})$ subsolution of (1.2) with $K(x) \equiv K_{0}$ in $\Omega$ for some $K_{0} \geq 0$, in a pioneering paper [4], C. Bandle proved that,

$$
L^{2}(\partial \Omega) \geq\left(4 \pi-\omega_{+}(\Omega)-K_{0} M(\Omega)\right) M(\Omega),
$$

where,

$$
L(\partial \Omega)=\int_{\partial \Omega} e^{\frac{f+u}{2}} d \ell, \text { and } M(\Omega)=\int_{\Omega} e^{f+u} d x .
$$

Here and in the rest of this paper $d \ell$ and $d x$ will denote the 1-dimensional and 2-dimensional Hausdorff measures respectively.

The inequality (1.3) is sharp and in [4] the case where the equality holds is characterized as well, see also [5]. Actually (1.3) admits a beautiful geometric interpretation in terms of the Alexandrov's isoperimetric inequality [2], as discussed in [4] and more extensively in [5]. As we will see later on, the original geometric setting of the problem in terms of singular isothermal coordinates [22], suggests that (1.3) should hold in a more general form. This is our motivation and indeed our main aim is to obtain a generalized version of (1.3) in a weak framework. To state our result, we need some definitions first.

Definition 1.1. We say that $E \subset \mathbb{R}^{2}$ is a simple domain, if it is an open and bounded domain whose boundary $\partial E$ is the support of a rectifiable Jordan curve. We will also say that $E \subset \mathbb{R}^{2}$ is a regular domain if it is a connected, open and bounded domain whose boundary $\partial E$ is the union of finitely many rectifiable Jordan curves.

Definition 1.2. Let $S \subset \Omega$ be a finite set. We say that

$$
f \in L_{\text {loc }}^{p, \text { loc }}(\Omega \backslash S) \text { or either } u \in W_{\text {loc }}^{2, p, \text { loc }}(\Omega \backslash S) \text {, for some } p>2 \text {, }
$$

if for each open and relatively compact set $U \Subset \Omega \backslash S$ there exists $p=p_{U}>2$ such that,

$$
f \in L^{p_{U}}(U) \text { or either } u \in W^{2, p_{U}}(U) .
$$

Also, by setting $B_{r}(S)=\bigcup_{p \in S} B_{r}(p)$, we say that,

$$
f \in L^{p \text {,loc }}(\Omega \backslash S) \text { or either } u \in W^{2, p, \text { loc }}(\Omega \backslash S) \text {, for some } p>2 \text {, }
$$

if for each $r>0$, there exists $p_{r}>2$ such that,

$$
f \in L^{p_{r}}\left(\Omega \backslash B_{r}(S)\right) \text { or either } u \in W^{2, p_{r}}\left(\Omega \backslash B_{r}(S)\right) .
$$

Definition 1.3. Let $f_{ \pm}$be two superharmonic functions in $\Omega$ taking the form (1.1), $u \in L_{\mathrm{loc}}^{1}(\Omega)$ and $K e^{f+u} \in L_{\mathrm{loc}}^{1}(\Omega)$. For any fixed and relatively compact Borel set $E \Subset \Omega$, we define,

$$
\mathcal{K}_{+}\left(E ; K_{0}\right)=\sup _{U \subseteq E}\left\{\frac{1}{2} \omega(U)+\int_{U}\left[K-K_{0}\right] e^{f+u} d x\right\},
$$


where the supremum is taken over all Borel sets $U \subseteq E$.

Finally we will need the following result about the local exponential integrability of $e^{f}$. Although similar exponential estimates for logarithmic potentials are well known, see [21] or more recently [11] and [31], it seems that the statement which is really needed here has been introduced only very recently in [1].

Proposition 1.4. Let $f_{ \pm}$be two superharmonic functions satisfying (1.1) in $\Omega$ and

$$
S_{2 \pi}=\left\{x \in \Omega: \omega_{+}(x) \geq 2 \pi\right\} .
$$

Then $S_{2 \pi}$ is finite, $d_{1}=\frac{1}{4} \operatorname{dist}\left(S_{2 \pi}, \partial \Omega\right)>0$ and we have:

(i) $e^{-\left(f_{-} h_{-}\right)} \in L^{\infty}(\Omega)$ and $e^{\left(f_{+}-h_{+}\right)} \in L^{p_{0}, \text { loc }}\left(\Omega \backslash S_{2 \pi}\right)$ for some $p_{0}>2$.

(ii) If

$$
\forall x \in \Omega, \omega_{+}(x)<4 \pi .
$$

holds, then $e^{\left(f_{+}-h_{+}\right)} \in L^{q_{0}}(\Omega)$ for some $q_{0}>1$.

Our main result is the following,

Theorem 1.5. Let $K_{0} \geq 0, f_{ \pm}$be two superharmonic functions taking the form (1.1) and satisfying (1.6), $q_{0}>1$ and $p_{0}>2$ be defined as in Proposition 1.4 and $K \in L_{\text {loc }}^{n \text { loc }}\left(\Omega \backslash S_{2 \pi}\right) \cap$ $L_{\text {loc }}^{s}(\Omega)$, for some $n>\frac{2 p_{0}}{p_{0}-2}$ and some $s>\frac{q_{0}}{q_{0}-1}$. Assume that, either,

(i) $K e^{f+u} \in L^{1}(\Omega)$, where $u \in L^{1}(\Omega)$ is a solution of (1.2) in the sense of distributions, or, (ii) $u \in W_{\text {loc }}^{2, p, \text { loc }}\left(\Omega \backslash S_{2 \pi}\right) \cap W_{\text {loc }}^{2, q}(\Omega)$, for some $p>2$ and some $q>1$, is a strong subsolution of (1.2), that is,

$$
-\Delta u \leq 2 K e^{f} e^{u} \quad \text { for a.a. } x \in \Omega .
$$

Then, if (i) holds, we have $u \in W_{\text {loc }}^{2, p, \text { loc }}\left(\Omega \backslash S_{2 \pi}\right) \cap W_{\text {loc }}^{2, q}(\Omega) \cap L_{\text {loc }}^{\infty}(\Omega)$, for some $p>2$ and some $q \in(1,2)$, and in particular $u$ is a strong solution of (1.2), that is, it satisfies (1.7) with the equality sign.

Moreover, in both cases, for any fixed simple and relatively compact subdomain $E \Subset \Omega$, we have $M(E)<+\infty$ and the following inequality holds:

$$
L^{2}(\partial E) \geq\left(4 \pi-2 \mathcal{K}_{+}\left(E ; K_{0}\right)-K_{0} M(E)\right) M(E) .
$$

The equality in (1.8) is attained if and only if $u$ is a strong solution of (1.2) in $E$ and,

$$
e^{f(z)+u(z)}=\frac{\tau^{2}\left|\Phi_{0}^{\prime}(z)\left(\Phi_{0}(z)\right)^{-\alpha}\right|^{2}}{\left(1+\frac{K_{0} \tau^{2}}{4(1-\alpha)^{2}}\left|\Phi_{0}(z)\right|^{2(1-\alpha)}\right)^{2}}, z \in E,
$$


for some $\tau \neq 0$, where $\alpha=\frac{1}{4 \pi} \omega_{+}(E)$ and $\Phi_{0}$ is a conformal map of $E$ onto the disk of unit radius, $\left|\Phi_{0}(z)\right|<1$ with $\Phi_{0}\left(z_{0}\right)=0$, for some $z_{0} \in E$.

(iii) If $\omega \perp e^{f+u} \mathcal{H}^{2}$, then the equality holds if and only if, in addition to the above conditions, one has $K \equiv K_{0}$ for a.a. $z \in E$ and $\omega=4 \pi \alpha \delta_{z=z_{0}}$, that is, $f(z)=h(z)+4 \pi \alpha G\left(z, z_{0}\right)=$ $h(z)-2 \alpha \log \left|\Phi_{0}(z)\right|$, for some harmonic function $h$ in $E$.

Remark 1.6. Let us denote by $\mathcal{H}^{2}$ the 2-dimensional Hausdorff measure. By using the fact that $\omega_{+} \perp \omega_{-}$, it is easy to check that if $\omega_{-} \perp e^{f+u} \mathcal{H}^{2}$, then we have,

$$
2 \mathcal{K}_{+}\left(E ; K_{0}\right)=\omega_{+}(E)+2 \int_{E}\left[K-K_{0}\right]^{+} e^{f+u} d x,
$$

where $K^{+}=\max \{K, 0\}$, while in general the equality sign should be replaced by the inequality sign.

As far as one is just concerned with the inequality and not with the characterization of the equality sign, then, in case (i) holds, (1.8) holds under much weaker conditions. The proof of this fact is based on Theorem 1.5] and on some results and arguments in [11] about the regularity properties of Liouville-type equations.

Corollary 1.7. Let $K_{0} \geq 0, f_{ \pm}$be two superharmonic functions taking the form (1.1) and satisfying (1.6), and $K \in L^{1}\left(\Omega ; e^{f+u} \mathcal{H}^{2}\right)$ where $u \in L^{1}(\Omega)$ is a solution of (1.2) in the sense of distributions. Then:

(i) $u \in W_{\text {loc }}^{1, r}(\Omega)$ for any $r \in(1,2)$ and $e^{t|u|} \in L_{\text {loc }}^{1}(\Omega)$ for any $t \geq 1$;

(ii) (1.8) holds.

Motivated by the study of a cosmic string equation, in a recent paper [8] we derived (1.8) in the easier situation where $\omega_{-} \equiv 0$ while $\omega_{+}$is proportional to a Dirac delta. The problem here is more subtle, and the crux of the proof is to attach to each strong subsolution of (1.2) an auxiliary function (which we will denote by $\eta$ ) which satisfies a Liouville type equation with Dirichlet boundary condition on $E$, and which admits a suitable locally absolutely continuous weighted rearrangement (which we will denote by $\eta^{*}$ ). The difficulty arises since, in view of the generality suggested by the geometric application, no assumption is made about $\omega$, with the unique exception of the "no-cusp" hypothesis (1.6). As a consequence, the term $e^{f_{+}}$, which is part of the weight factor in the weighted rearrangement, can come with almost any kind of singularity. In particular, the standard argument [25] yielding the absolute continuity of $\eta^{*}$, does not work in this case, neither in the slightly improved form used to handle conical singularities, see [8]. We succeed in solving this problem by a careful decomposition of the singular set of $\omega_{+}$, see the definition of $S_{2 \pi}$ in (1.5). The point is that $S_{2 \pi}$ is finite in $\Omega$, while, locally in its complement, we come up with enough summability for $e^{f}$ to guarantee that $\eta^{*}$ is absolutely continuous. This 
approach, recently pursued in [1] to prove a regularity result for a class of singular surfaces introduced by Alexandrov [3], motivates the peculiar notations introduced in Definition [1.2, In particular, the assumptions about $K$ and $u$, are essentially the minimal requirements to match the regularity of $\eta$ as allowed by the properties of $f$ derived in this way. It is understood that the characterization of the equality case in this weak contest is new as well.

The proof of Theorem 1.5 is split into four steps. In the first and second step we construct $\eta$, its weighted rearrangement $\eta^{*}$ and prove that $\eta^{*}$ is locally absolutely continuous. Step four contains the discussion about the equality case. Step three is the adaptation in our setting of the part of the Bandle argument which is concerned with the derivation of a differential inequality and its consequences.

In the second part of this paper, and in the same spirit of [4], we will apply (1.8) to derive a new proof of the Alexandrov isoperimetric inequality for $K_{0} \geq 0$ on abstract surfaces of bounded curvature, see (4.9) in Theorem 4.7. We refer the reader to [5], [6], [13], [17], [19] and the references therein for a detailed exposition of the proof and of the interesting history of Alexandrov's inequality and to [26, 27] for other more recent proofs. See also [9], 23]. While in the above references one can find various proofs of the inequality (4.9), we were not able to find a proof of the characterization of the equality case in the weak context pursued here, which seems therefore to be new even in the geometric setting.

Besides, to apply our estimates to this problem, we need to prove a seemingly new characterization of the structure of the metrics in local isothermal coordinates for certain classes of singular surfaces, see Theorem 4.4. This intermediate result can also be seen as another result in the description of the regularity properties of isothermal coordinates systems on Alexandrov's surfaces of bounded curvature recently pursued in [1. Finally, some explicit examples are discussed to illustrate these results, including the isoperimetric inequality (4.9) on various singular surfaces homeomorphic to the 2-sphere.

We conclude this introduction with a remark about the case where $E$ is not simple but just regular, that is, the possibility that $E$ could be connected but not simply connected.

Remark 1.8. If $\Omega$ is simply connected and the assumptions of Theorem 1.5 are satisfied, but if the set $E \Subset \Omega$ is just assumed to be regular, then it is straightforward to check that our proof yields the following inequality,

$$
L^{2}(\partial E)>\left(4 \pi-2 \mathcal{K}_{+}\left(E_{s} ; K_{0}\right)-K_{0} M(E)\right) M(E)
$$


D.B. \& D.C.

where $E_{s}$ is the interior of the closure of the union of $E$ with the bounded components of $\mathbb{R}^{2} \backslash E$ (the "holes" of $E$ ), which we denote by $(E)_{B}$, that is,

$$
E_{s}=\frac{\circ}{E \cup(E)_{B}}
$$

In other words we still have an inequality of the form (1.8), but we have a worse isoperimetric ratio, which is essentially obtained by subtracting the terms of the total curvature relative to the "holes" of E. This is not a technical point, and in fact it is possible to construct counterexamples to the inequality where these terms are omitted, see for example p.14 in [9]. The proof of this inequality is really the same as that of Theorem 1.5, but for the fact that in (3.22) and in (3.23) below we use the Huber inequality (2.2) for the non contractible domain E. In particular this is also why we obtain the strict inequality in this case. It is straightforward to check that if the assumptions of Corollary 1.7 are satisfied, then (1.10) holds with the $\geq$ sign replacing the strict inequality.

This paper is organized as follows. In section 2 we prove Proposition 1.4 and discuss the Huber's inequality. In section 3 we prove Theorem 1.5 and Corollary 1.7. Sections 4 and 5 are devoted to the discussion of the Alexandrov's isoperimetric inequality and related examples.

\section{Preliminary estimates: exponential Summability of SUbHarmonic FunCtions AND Huber's INEQUALity.}

The local exponential integrability of $e^{f_{+}}$as claimed in Proposition 1.4 is not new, see [1. We provide the proof of Proposition 1.4 for the sake of completeness.

The Proof of Proposition 1.4.

We will denote by $d_{\Omega}$ the diameter of $\Omega$. Clearly $S_{2 \pi}$ is finite since $\omega_{+}$is finite, whence obviously $\operatorname{dist}\left(S_{2 \pi}, \partial \Omega\right)>0$. Let $d_{1}=\frac{1}{4} \operatorname{dist}\left(S_{2 \pi}, \partial \Omega\right)$ and let us set $\Omega_{d}=\{x \in \Omega: \operatorname{dist}(x, \partial \Omega)<d\}$. Then $\omega_{+}\left(\Omega_{d}\right) \searrow 0^{+}$, as $d \searrow 0^{+}$, whence there exists $d_{0}>0$ such that $\omega^{+}\left(\Omega_{d}\right)<\frac{\pi}{2}$, for each $d<4 d_{0}$. We choose $d_{0}$ possibly smaller to satisfy $4 d_{0}<d_{1}$. It is not difficult to see that there exists $C_{0}>0$ such that,

$$
\left(f_{+}(x)-h_{+}(x)\right)-C_{0} \leq w_{0}(x):=\frac{1}{2 \pi} \int_{\Omega_{2 d_{0}}} \log \left(\frac{d_{\Omega}}{|x-y|}\right) d \omega_{+}, \forall x \in \Omega_{d_{0}} .
$$

By the Jensen's inequality and the Fubini-Tonelli Theorem we can estimate,

$$
\int_{\Omega_{d_{0}}} \exp \left(\frac{3 \pi w_{0}}{\omega_{+}\left(\Omega_{2 d_{0}}\right)}\right) d x \leq
$$




$$
\begin{gathered}
\int_{\Omega_{d_{0}}} d x \int_{\Omega_{2 d_{0}}}\left(\frac{d_{\Omega}}{|x-y|}\right)^{\frac{3}{2}} \frac{d \omega_{+}(y)}{\omega_{+}\left(B_{2 d_{0}}\right)}=\int_{\Omega_{2 d_{0}}} \frac{d \omega_{+}(y)}{\omega_{+}\left(\Omega_{2 d_{0}}\right)} \int_{\Omega_{d_{0}}}\left(\frac{d_{\Omega}}{|x-y|}\right)^{\frac{3}{2}} d x \leq \\
\int_{\Omega_{2 d_{0}}} \frac{d \omega_{+}(y)}{\omega_{+}\left(\Omega_{2 d_{0}}\right)} \int_{B_{d_{\Omega}}(y)}\left(\frac{d_{\Omega}}{|x-y|}\right)^{\frac{3}{2}} d x=\pi\left(2 d_{\Omega}\right)^{2},
\end{gathered}
$$

where we used the fact that $\Omega_{d_{0}} \subset B_{d_{\Omega}}(y)$. This inequality shows that $e^{\left(f_{+}-h_{+}\right)} \in L^{6}\left(\Omega_{d_{0}}\right)$.

(i) Since $-\left(f_{-}-h_{-}\right)$is negative, then $e^{-\left(f_{--} h_{-}\right)} \in L^{\infty}(\Omega)$. Let $\Omega_{0}=\left\{\Omega \backslash \Omega_{\frac{d_{0}}{2}}\right\} \backslash B_{r}\left(S_{2 \pi}\right)$, with $0<r<d_{1}$, and let us fix $x_{0} \in \overline{\Omega_{0}}$. Since $\omega_{+}\left(x_{0}\right)<2 \pi$, then we can find $\varepsilon>0$ such that there exists $R>0$ depending on $x_{0}$ and $\varepsilon$, such that the ball centred at $x_{0}, B_{2 R}:=B_{2 R}\left(x_{0}\right)$, satisfies $B_{2 R} \Subset\left\{\Omega \backslash \Omega_{d_{0}}\right\} \backslash S_{2 \pi}$ and $\omega_{+}\left(B_{2 R}\right) \leq 2 \pi-2 \varepsilon$. As above there exists $C>0$ such that,

$$
\left(f_{+}(x)-h_{+}(x)\right)-C \leq w(x):=\frac{1}{2 \pi} \int_{B_{2 R}} \log \left(\frac{4 R}{|x-y|}\right) d \omega_{+}, \forall x \in D_{x_{0}} \equiv D_{0}:=B_{R}\left(x_{0}\right),
$$

and for any $\delta<4 \pi$ we can estimate,

$$
\begin{gathered}
\int_{D_{0}} \exp \left(\frac{(4 \pi-\delta) w}{\omega_{+}\left(B_{2 R}\right)}\right) d x \leq \\
\int_{D_{0}} d x \int_{B_{2 R}}\left(\frac{d_{\Omega}}{|x-y|}\right)^{2-\frac{\delta}{2 \pi}} \frac{d \omega_{+}(y)}{\omega_{+}\left(B_{2 R}\right)}=\int_{B_{2 R}} \frac{d \omega_{+}(y)}{\omega_{+}\left(B_{2 R}\right)} \int_{D_{0}}\left(\frac{d_{\Omega}}{|x-y|}\right)^{2-\frac{\delta}{2 \pi}} d x \leq \\
\int_{B_{2 R}} \frac{d \omega_{+}(y)}{\omega_{+}\left(B_{2 R}\right)} \int_{B_{d_{\Omega}}(y)}\left(\frac{d_{\Omega}}{|x-y|}\right)^{2-\frac{\delta}{2 \pi}} d x=\frac{\left(2 \pi d_{\Omega}\right)^{2}}{\delta} .
\end{gathered}
$$

Therefore, in particular by choosing $\delta<\varepsilon$, we see that $p_{D_{0}}:=\frac{(4 \pi-\delta)}{\omega_{+}\left(D_{0}\right)}>\frac{(4 \pi-\varepsilon)}{\omega_{+}\left(B_{2 R}\right)}>2$ so that $e^{\left(f_{+}-h_{+}\right)} \in L^{p_{D_{0}}}\left(D_{0}\right)$, for some $p_{D_{0}}>2$ depending on $x_{0}$ and $R$.

At this point we define $\mathcal{B}=\bigcup_{x \in \overline{\Omega_{0}}} D_{x}$, where each $D_{x}$, constructed as above, comes with its own $p_{D_{x}}>2$. Clearly $\mathcal{B}$ is an open cover of $\overline{\Omega_{0}}$, and since $\overline{\Omega_{0}}$ is compact, then we can extract a finite cover $D_{x_{j}}, j=1, \ldots, N$, and set $p_{U}:=\min \left\{6, \min _{j=1, . ., N} p_{D_{x_{j}}}\right\}$. Therefore $e^{\left(f_{+}-h_{+}\right)} \in$ $L^{p_{U}}\left(\Omega \backslash B_{r}\left(S_{2 \pi}\right)\right)$, for some $p_{U}>2$, which proves $(i)$.

(ii) Let us define $\Omega_{1}=\Omega \backslash \Omega_{\frac{d_{0}}{2}}$. We use (1.6), as in the proof of $(i)$ to conclude that $e^{\left(f_{+}-h_{+}\right)} \in$ $L^{q}\left(\Omega_{1}\right)$ for some $q>1$. Therefore we find $e^{\left(f_{+}-h_{+}\right)} \in L^{q_{0}}(\Omega)$ where $q_{0}=\min \{6, q\}>1$, as claimed.

Next we present the well known Huber's inequality [18] as well as a generalization of it suitable to be applied to regular (whence in particular non simply connected) domains. 
Theorem 2.1 (The Huber inequality, [18). Let $\Omega \subset \mathbb{R}^{2}$ be open and bounded and $E \Subset \Omega$ be a simple and relatively compact subset. Let $f$ be the difference of two superharmonic functions in $\Omega$ taking the form (1.1). Then it holds,

$$
\left(\int_{\partial E} e^{\frac{f}{2}} d \ell\right)^{2} \geq\left(4 \pi-\omega_{+}(E)\right) \int_{E} e^{f} d x .
$$

The equality holds in (2.1) if and only if, in complex notations, $f(z)=c+2 \log \left|\Phi^{\prime}(z)\left(\Phi(z)^{-\alpha_{E}}\right)\right|$ where $\alpha_{E}=\frac{1}{4 \pi} \omega_{+}(E)$ and $\Phi$ is a conformal map of $E$ onto the disk of unitary radius $|w|=$ $|\Phi(z)|<1$ with $\Phi\left(z_{0}\right)=0$ for some $z_{0} \in E$.

We will need the following generalization of the Huber's result.

Theorem 2.2. Let $\Omega \subset \mathbb{R}^{2}$ be open and bounded and $E \Subset \Omega$ be a simple and relatively compact subset. Let $f$ be the difference of two superharmonic functions in $\Omega$ taking the form (1.1). If $U \subseteq E$ is a regular domain, then it holds,

$$
\left(\int_{\partial U} e^{\frac{f}{2}} d \ell\right)^{2} \geq\left(4 \pi-\omega_{+}(E)\right) \int_{U} e^{f} d x .
$$

In particular, if $U$ is not simply connected, then the inequality is strict.

Proof. In view of Theorem 2.1 we are left to discuss the cases where $U$ is not simply connected and prove in particular that in all those cases the inequality is strict. Obviously the inequality is trivially satisfied if $\omega_{+}(E) \geq 4 \pi$, whence we assume w.l.o.g. that $\omega_{+}(E)<4 \pi$. Let us assume for the moment that $U=U_{1} \backslash \overline{U_{0}}$ for a pair of simple domains such that $U_{0} \Subset U_{1}$ and $\partial U=$ $\partial U_{1} \cup \partial U_{0}$. So $U_{1}=U \cup \overline{U_{0}}$ and in this case, by assumption we have $E=U_{1}$ and in particular $\omega_{+}\left(U_{0}\right)<\omega_{+}\left(U_{1}\right)<4 \pi$. For any domain $U \subset \mathbb{R}^{2}$, let us set

$$
\ell(\partial U)=\int_{\partial U} e^{\frac{f}{2}} d \ell, \quad M(U)=\int_{U} e^{f} d x .
$$

Thus we may use (2.1) to obtain

$$
\begin{gathered}
\ell^{2}(\partial U)=\ell^{2}\left(\partial U_{1} \cup \partial U_{0}\right)>\ell^{2}\left(\partial U_{1}\right)+\ell^{2}\left(\partial U_{0}\right) \geq \\
\left(4 \pi-\omega_{+}\left(U_{1}\right)\right) M\left(U_{1}\right)+\left(4 \pi-\omega_{+}\left(U_{0}\right)\right) M\left(U_{0}\right)> \\
\left(4 \pi-\omega_{+}\left(U_{1}\right)\right) M\left(U_{1}\right)>\left(4 \pi-\omega_{+}\left(U_{1}\right)\right) M(U),
\end{gathered}
$$


which is (2.2) in this particular case. The case where $\mathbb{R}^{2} \backslash U$ has finitely many bounded components readily follows by an induction argument on the number of "holes" of $U$. Obviously the inequality is always strict whenever $U$ is not simply connected.

\section{The proof of Theorem 1.5 and Corollary 1.7.}

This section is devoted to the proof of Theorem 1.5 and Corollary 1.7 .

\section{The proof of Theorem 1.5.}

Once the result has been established for $K_{0} \neq 0$, then the case $K_{0}=0$ is worked out by an elementary limiting argument, which is why we will just discuss the case $K_{0}>0$.

We recall that by assumption $\frac{n p_{0}}{n+p_{0}}>2$ and $\frac{s q_{0}}{s+q_{0}}>1$. First of all, we have the following,

Lemma 3.1. (a) If (i) holds and if $K \in L_{\mathrm{loc}}^{s}(\Omega)$ for some $s>\frac{q_{0}}{q_{0}-1}$, then,

$$
K e^{f+u} \in L_{\text {loc }}^{r}(\Omega),
$$

and $u \in L_{\mathrm{loc}}^{\infty}(\Omega) \cap W_{\mathrm{loc}}^{2, r}(\Omega)$, for any $1 \leq r \leq \frac{s q_{0}}{s+q_{0}}$. In particular $u$ is a strong solution of (1.2).

(b) If $(i)$ holds and if $K \in L_{\text {loc }}^{n \text {,loc }}\left(\Omega \backslash S_{2 \pi}\right) \cap L_{\text {loc }}^{s}(\Omega)$ for some $n>\frac{2 p_{0}}{p_{0}-2}$ and some $s>\frac{q_{0}}{q_{0}-1}$, then,

$$
K e^{f+u} \in L_{\text {loc }}^{k, \text { loc }}\left(\Omega \backslash S_{2 \pi}\right) \cap L_{\text {loc }}^{r}(\Omega),
$$

and $u \in W_{\mathrm{loc}}^{2, k, \mathrm{loc}}\left(\Omega \backslash S_{2 \pi}\right) \cap W_{\mathrm{loc}}^{2, r}(\Omega) \cap L_{\mathrm{loc}}^{\infty}(\Omega)$, for any $2<k \leq \frac{n p_{0}}{n+p_{0}}$ and $1 \leq r \leq \frac{s q_{0}}{s+q_{0}}$. In particular $u$ is a strong solution of (1.2).

Proof. (a) By assumption we have $\frac{s q_{0}}{s+q_{0}}>1$ and then, in view of Proposition 1.4, we also have $K e^{f} \in L_{\text {loc }}^{q}(\Omega), \quad \forall 1<q \leq \frac{s q_{0}}{s+q_{0}}$. On the other hand, since $K e^{f+u} \in L^{1}(\Omega)$, then, by Remark 2 in [11], we have $e^{|u|} \in L_{\text {loc }}^{k}(\Omega)$ for any $k>0$, and therefore in particular $e^{u} \in L^{q^{\prime}}(\Omega)$, where $q^{\prime}=\frac{q}{q-1}<+\infty$. Thus we can apply another result in [11] (see Remark 5 in [11]), which yields $u \in L_{\mathrm{loc}}^{\infty}(\Omega)$. So, by standard elliptic estimates, we conclude also that $u \in W_{\mathrm{loc}}^{2, r}(\Omega)$ and in particular that $u$ is a strong solution of (1.2).

(b) Next, let us fix a compact set $U \subset \Omega \backslash S_{2 \pi}$ and observe that, by assumption, $K \in L^{n}(U)$ for some $n>\frac{2 p_{U}}{p_{U}-2}$. Therefore $\frac{n p_{U}}{n+p_{U}}>2$ and then, in view of Proposition 1.4, we also have $K e^{f} \in L^{p}(U), \forall 2<p \leq \frac{n p_{U}}{n+p_{U}}$. Since $U$ is arbitrary, then we conclude that $K e^{f+u} \in L_{\text {loc }}^{k \text {,loc }}(\Omega \backslash$ $\left.S_{2 \pi}\right) \cap L_{\text {loc }}^{r}(\Omega)$, for any $2<k \leq \frac{n p_{U}}{n+p_{U}}$ and $1<r \leq \frac{s q_{0}}{s+q_{0}}$. As above, by standard elliptic estimates, we conclude also that $u \in W_{\text {loc }}^{2, k \text { loc }}\left(\Omega \backslash S_{2 \pi}\right) \cap W_{\text {loc }}^{2, r}(\Omega)$ and in particular that $u$ is a strong solution of (1.2). 
Lemma 3.1 shows that if $(i)$ holds, then $u \in W_{\text {loc }}^{2, p, \text { loc }}\left(\Omega \backslash S_{2 \pi}\right) \cap W_{\text {loc }}^{2, q}(\Omega) \cap L_{\text {loc }}^{\infty}(\Omega)$, for some $p>2$ and $q>1$, and moreover that $u$ is a strong solution of (1.2). Whence we are reduced to the analysis of the case where $u \in W_{\text {loc }}^{2, p \text { loc }}\left(\Omega \backslash S_{2 \pi}\right) \cap W_{\text {loc }}^{2, q}(\Omega) \cap L_{\text {loc }}^{\infty}(\Omega)$, for some $p>2$ and $q>1$, satisfies (1.7). In particular, in the rest of the proof, we will use the fact that, by the Sobolev embedding Theorem, $u \in C_{\mathrm{loc}}^{0}(\Omega)$. Clearly, in view of (3.1), $M(E)$ is finite. We divide the proof into four steps.

\section{Step 1.}

Since $E \Subset \Omega$ is relatively compact and simple, then we can find an open, simply connected, relatively compact and smooth domain $\Omega_{0}$ such that,

$$
E \Subset \Omega_{0} \Subset \Omega .
$$

Since $S_{2 \pi}$ is finite and since $\omega_{ \pm}\left(\Omega_{0}\right)<+\infty$, then we can choose $\Omega_{0}$ such that, for some $N \in \mathbb{N}$,

$$
S_{2 \pi}^{0}:=S_{2 \pi} \cap \Omega_{0}=\left\{q_{1}, \ldots, q_{N}\right\} \subset \Omega_{0} \text { and } \partial \Omega_{0} \cap S_{2 \pi}=\emptyset .
$$

Clearly, in view of (1.7), we have,

$$
-\Delta u \leq 2 K e^{f} e^{u}=2\left[K-K_{0}\right] e^{f} e^{u}+2 K_{0} e^{f} e^{u} \text { for a.a. } x \in \Omega_{0} .
$$

Next, let us define,

$$
\phi(x):=-\Delta u-2\left[K-K_{0}\right] e^{f} e^{u}-2 K_{0} e^{f} e^{u}, \quad x \in \Omega_{0} .
$$

Since $u \in W_{\text {loc }}^{2, p \text { loc }}\left(\Omega \backslash S_{2 \pi}\right) \cap W^{2, q}(\Omega) \cap C_{\text {loc }}^{0}(\Omega)$, for some $p>2$ and some $q>1$, and in view of (3.1) and of Proposition 1.4, we see from (3.3) that,

$$
\phi(x) \leq 0, \quad \text { for a.a. } x \in \Omega_{0} \quad \text { and } \quad \phi \in L_{\text {loc }}^{p \text { loc }}\left(\Omega_{0} \backslash S_{2 \pi}^{0}\right) \cap L^{q}\left(\Omega_{0}\right),
$$

for some $p>2$ and some $q>1$. Therefore, in view of Theorem 9.15, Corollary 9.18 and Lemma 9.17 in [16] we see that the linear problem,

$$
\Delta w=\phi \text { in } \Omega_{0}, \quad w=0 \text { on } \partial \Omega_{0},
$$

admits a unique strong solution $w \in W_{\text {loc }}^{2, p \text { loc }}\left(\Omega_{0} \backslash S_{2 \pi}^{0}\right) \cap W^{2, q}\left(\Omega_{0}\right) \cap C^{0}\left(\overline{\Omega_{0}}\right)$, for some $p>2$ and some $q>1$. Obviously $w$ is superharmonic (see [16] $\S 2.8$ and Ex. 2.7, 2.8).

Next let $f_{1}$ be the Perron's (see $\S 2.8$ in [16]) solution of $\Delta f_{1}=0$ in $E, f_{1}=-u$ on $\partial E$. Since $u \in C^{0}(\bar{E})$, then $f_{1}$ is well defined and continuous up to the boundary (see $\S 2.8$ in [16]). Let us also define $f_{2}$ to be the unique $W_{\text {loc }}^{2, p \text { loc }}\left(\Omega_{0} \backslash S_{2 \pi}^{0}\right) \cap W^{2, q}\left(\Omega_{0}\right) \cap C^{0}\left(\overline{\Omega_{0}}\right)$ (for some $p>2$ and some $q>1$ ) solution of the linear problem,

$$
-\Delta f_{2}=2\left[K-K_{0}\right] e^{f} e^{u} \text { in } \Omega_{0}, \quad f_{2}=0 \text { on } \partial \Omega_{0} .
$$


With these definitions, we may finally set $\eta=u+w+f_{1}-f_{2}$. Then, we see that $\eta \in W_{\text {loc }}^{2, p, \text { loc }}(E \backslash$ $\left.S_{2 \pi}^{0}\right) \cap W^{2, q}(E) \cap C^{0}(\bar{E})$ for some $p>2$ and some $q>1$ and satisfies,

$$
-\Delta \eta=2 K_{0} e^{\psi} e^{\eta} \text { for a.a. } x \in E, \quad \eta=0 \text { on } \partial E,
$$

where

$$
\psi=f_{+}+f_{2}-f_{-}-w-f_{1} .
$$

By the Sobolev embedding Theorem we conclude that,

$$
\eta_{+} \in C_{\mathrm{loc}}^{1}\left(E \backslash S_{2 \pi}^{0}\right) .
$$

Since $\eta \in W^{2, q}(E)$, for some $q>1$, then by using the Sobolev embedding once more we see that $\eta \in W^{1,2}(E) \cap C^{0}(E)$. Then by the maximum principle for weak solutions (see for example Theorem 8.1 in [16]) we deduce that $\eta \geq 0$. In particular, by the strong maximum principle for weak supersolutions (see for example Theorem 8.18 in [16]) we also check that $\eta$ is strictly positive in $E$. In particular, we conclude that,

$$
\eta(x)>0 \quad \forall x \in E \text { and } \eta(x)=0 \Longleftrightarrow x \in \partial E .
$$

\section{Step 2.}

Let us set $t_{+}=\max _{\bar{E}} \eta$,

$$
d \tau=e^{\psi} d x, \quad \sigma=e^{\frac{\psi}{2}} d \ell
$$

and let us define,

$$
\Omega(t)=\{x \in E \mid \eta(x)>t\}, \quad t \in\left[0, t_{+}\right), \quad \Gamma(t)=\{x \in E \mid \eta(x)=t\}, \quad t \in\left[0, t_{+}\right],
$$

and

$$
\mu(t)=\int_{\Omega(t)} d \tau .
$$

Since $\eta$ satisfy (3.6), then $\Gamma(t)$ has null measure, whence we conclude that $\mu$ is continuous. Moreover, in view of (3.9), we find that,

$$
\Omega(0)=E, \quad \Gamma(0)=\partial E, \quad \mu(0)=\int_{E} d \tau .
$$

Clearly we can extend $\mu$ on $\left[0, t_{+}\right]$by setting $\mu\left(t_{+}\right)=\lim _{t \nearrow t_{+}} \mu(t)=0^{+}$, whence $\mu \in C^{0}\left(\left[0, t_{+}\right]\right)$. Next, by using (3.6) once more, it is not difficult to see that the 2-dimensional measure of the set $\{x \in E: \nabla \eta(x)=0\}$ vanishes. Therefore, by a well known consequence of the co-area formula 
(see for example [12 p.158) and of the Sard's Lemma for Sobolev functions [14] (here we use also (3.2)), we see that

$$
\frac{d \mu(t)}{d t}=-\int_{\Gamma(t)} \frac{e^{\psi}}{|\nabla \eta|} d \ell
$$

for a.a. $t \in\left[0, t_{+}\right]$.

At this point, for any $s \in[0, \mu(0)) \equiv\left[\mu\left(t_{+}\right), \mu(0)\right)$, we introduce a weighted rearrangement of $\eta$,

$$
\eta^{*}(s)=\left|\left\{t \in\left[0, t_{+}\right]: \mu(t)>s\right\}\right|,
$$

where $|U|$ denotes the Lebesgue measure of a Borel set $U \subset \mathbb{R}$. By setting $\eta^{*}(\mu(0))=0$, then $\eta^{*} \in C^{0}([0, \mu(0)])$ is the inverse of $\mu$ on $\left[0, t_{+}\right]$and coincides with the distribution function of $\mu$. Actually $\eta^{*}$ is strictly decreasing, whence differentiable almost everywhere. A crucial point at this stage is to prove that $\eta^{*}$ is not just continuous and differentiable almost everywhere, but also locally absolutely continuous. It turns out that in fact it is locally Lipschitz in $(0, \mu(0))$ as shown in the following Lemma:

Lemma 3.2. For any $0<\bar{a} \leq a<b \leq \bar{b}<\mu(0)$, there exist $\bar{C}=\bar{C}\left(\bar{a}, \bar{b}, S_{2 \pi}, \mathcal{K}_{+}\left(E ; K_{0}\right)\right)>0$ such that,

$$
\eta^{*}(a)-\eta^{*}(b) \leq \bar{C}(b-a) .
$$

Proof. In view of (3.2) and (3.8), we see that $|\nabla \eta| \leq C_{U}$ on any $U \Subset E \backslash S_{2 \pi}^{0}$. Let us then set $t_{i}=\eta\left(x_{i}\right), x_{i} \in S_{2 \pi}, i=1, . ., m$, with $m \leq N$, and $t_{0}=\eta^{*}(a)$ and $t_{m+1}=\eta^{*}(b)$. For any

$$
\varepsilon<\min \left\{\frac{\left|\eta^{*}(a)-\eta^{*}(b)\right|}{4(m+1)}, \frac{1}{4} \min _{i=0, \ldots, m}\left\{t_{i+1}-t_{i}\right\}\right\},
$$

we can find $\delta=\delta_{\varepsilon}$ such that $\eta^{-1}\left[t_{i}+\varepsilon, t_{i+1}-\varepsilon\right] \cap B_{\delta}\left(S_{2 \pi}\right)=\emptyset$ for any $i=0, . ., m$, where $B_{\delta}\left(S_{2 \pi}\right)$ is a $\delta$-neighbourhood of the set $S_{2 \pi}$. Therefore, in particular, we can find $C_{\varepsilon}>0$ such that $|\nabla \eta(x)| \leq C_{\varepsilon}, \forall x \in \eta^{-1}\left[t_{i}+\varepsilon, t_{i+1}-\varepsilon\right]$. At this point, since $K_{0}>0$, then we can assume w.l.o.g. that $2 \gamma_{E}\left(K_{0}\right):=4 \pi-2 \mathcal{K}_{+}\left(E ; K_{0}\right)>0$ (otherwise $4 \pi-2 \mathcal{K}_{+}\left(E ; K_{0}\right)-K_{0} M(E)<0$ and (1.8) would be trivially satisfied). Therefore we can use the coarea formula (see [12] p. 158) and the Huber's isoperimetric inequality (2.2), to conclude that,

$$
\begin{gathered}
b-a=\mu\left(\eta^{*}(b)\right)-\mu\left(\eta^{*}(a)\right)=\int_{\eta>\eta^{*}(b)} d \tau-\int_{\eta>\eta^{*}(a)} d \tau=\int_{\eta^{*}(b)<\eta \leq \eta^{*}(a)} d \tau \geq \\
\int_{\eta^{*}(b)<\eta<\eta^{*}(a)} d \tau=\int_{\eta^{*}(b)}^{\eta^{*}(a)}\left(\int_{\Gamma(t)} \frac{d \sigma}{|\nabla \eta|}\right) d t=\sum_{i=0}^{m} \int_{t_{i}}^{t_{i+1}}\left(\int_{\Gamma(t)} \frac{d \sigma}{|\nabla \eta|}\right) d t \geq
\end{gathered}
$$




$$
\begin{gathered}
\sum_{i=0}^{m} \int_{t_{i}+\varepsilon}^{t_{i+1}-\varepsilon}\left(\int_{\Gamma(t)} \frac{d \sigma}{|\nabla \eta|}\right) d t \geq \frac{1}{C_{\varepsilon}} \sum_{i=0}^{m} \int_{t_{i}+\varepsilon}^{t_{i+1}-\varepsilon}\left(\int_{\Gamma(t)} d \sigma\right) d t \geq \\
\frac{\sqrt{2 \gamma_{E}\left(K_{0}\right)}}{C_{\varepsilon}} \sum_{i=0}^{m} \int_{t_{i}+\varepsilon}^{t_{i+1}-\varepsilon} \sqrt{\left(\int_{\Omega(t)} d \tau\right)} \geq \frac{\sqrt{2 \gamma_{E}\left(K_{0}\right)}}{C_{\varepsilon}} \sqrt{\left(\int_{\Omega\left(\eta^{*}(\bar{b})\right)} d \tau\right)} \sum_{i=0}^{m} \int_{t_{i}+\varepsilon}^{t_{i+1}-\varepsilon} d t= \\
C\left(\bar{a}, \bar{b}, S_{2 \pi}, \mathcal{K}_{+}\left(E ; K_{0}\right)\right)\left|\eta^{*}(a)-\eta^{*}(b)-2(m+1) \varepsilon\right| \geq \frac{1}{4} C\left|\eta^{*}(a)-\eta^{*}(b)\right|,
\end{gathered}
$$

for a strictly positive constant $C$ depending on $\bar{a}, \bar{b}, S_{2 \pi}, \mathcal{K}_{+}\left(E ; K_{0}\right)$, as claimed.

\section{Step 3.}

In view of (3.11) we obtain,

$$
\frac{d \eta^{*}(s)}{d s}=-\left(\int_{\Gamma\left(\eta^{*}(s)\right)} \frac{e^{\psi}}{|\nabla \eta|} d \ell\right)^{-1},
$$

for any $s \in I^{*}$, where $[0, \mu(0)] \backslash I^{*}$ is a set of null measure and, by setting $I:=\eta^{*}\left(I^{*}\right)$, then $\mu(I)=I^{*}$. Next, let us define,

$$
F(s)=2 K_{0} \int_{\Omega\left(\eta^{*}(s)\right)} e^{\eta} d \tau, \quad s \in[0, \mu(0)],
$$

where,

$$
F(\mu(0))=2 K_{0} \int_{E} e^{\eta} d \tau=2 K_{0} M(E)
$$

and we have set,

$$
F(0)=\lim _{s \searrow 0^{+}} F(s)=0^{+}
$$

Clearly $F(s)$ is strictly increasing and continuous on $[0, \mu(0)]$ and in particular locally Lipschitz in $(0, \mu(0))$, since in fact it satisfies,

$$
\left|F(s)-F\left(s_{0}\right)\right| \leq C\left|\mu\left(\eta^{*}(s)\right)-\mu\left(\eta^{*}\left(s_{0}\right)\right)\right|=C\left|s-s_{0}\right|, \quad \forall 0=\mu\left(t_{+}\right)<s_{0}<s<\mu(0),
$$

for a suitable constant $C>0$. In particular it holds,

$$
\int_{\Omega\left(\eta^{*}(s)\right)} e^{u} d \tau=\int_{0}^{s} e^{\eta^{*}(\lambda)} d \lambda, \quad \forall s \in[0, \mu(0)],
$$


so that,

$$
\frac{d F(s)}{d s}=2 K_{0} e^{\eta^{*}(s)}, \quad \frac{d^{2} F(s)}{d s^{2}}=2 K_{0} \frac{d \eta^{*}(s)}{d s} e^{\eta^{*}(s)}=\frac{d \eta^{*}(s)}{d s} \frac{d F(s)}{d s}, \quad \forall s \in I^{*} .
$$

We remark that since $\eta^{*}(s)$ is differentiable almost everywhere, then the formula for the first derivative of $F(s)$ shows that in fact $\frac{d F(s)}{d s}$ is differentiable almost everywhere as well. For any $s \in I^{*}$ the Cauchy-Schwartz inequality yields,

$$
\begin{gathered}
\left.\left(\int_{\Gamma\left(\eta^{*}(s)\right)} d \sigma\right)^{2} \leq \int_{\Gamma\left(\eta^{*}(s)\right)} \frac{e^{\psi}}{|\nabla \eta|} d \ell\right)\left(\int_{\Gamma\left(\eta^{*}(s)\right)}|\nabla \eta| d \ell\right)= \\
\left(-\frac{d \eta^{*}(s)}{d s}\right)^{-1}\left(\int_{\Gamma\left(\eta^{*}(s)\right)}\left(-\frac{\partial \eta}{\partial \nu_{+}}\right) d \ell\right),
\end{gathered}
$$

where $\nu_{+}=\frac{\nabla \eta}{|\nabla \eta|}$ is the exterior unit normal to $\Omega\left(\eta^{*}(s)\right)$ and we have used (3.14). Obviously, we can assume w.l.o.g. that $\eta^{-1}\left(S_{2 \pi} \cap E\right) \notin I$, so that, since $\eta$ satisfies (3.8), then (3.6) readily implies that,

$$
\int_{\Gamma\left(\eta^{*}(s)\right)}\left(-\frac{\partial \eta}{\partial \nu_{+}}\right) d \ell=\int_{\Omega\left(\eta^{*}(s)\right)} 2 K_{0} e^{\eta} d \tau,
$$

for any $s \in I^{*}$. Therefore, in particular we deduce that,

$$
\int_{\left.\Gamma\left(\eta^{*}(s)\right)\right)}\left(-\frac{\partial \eta}{\partial \nu_{+}}\right) d \ell=\int_{\Omega\left(\eta^{*}(s)\right)} 2 K_{0} e^{\eta} d \tau=F(s),
$$

for any $s \in I^{*}$. Plugging this identity in (3.18) we find,

$$
\left(\int_{\Gamma\left(\eta^{*}(s)\right)} d \sigma\right)^{2} \leq\left(-\frac{d \eta^{*}(s)}{d s}\right)^{-1} F(s),
$$

for any $s \in I^{*}$. Clearly, in view of (3.7), we have,

$$
-\Delta \psi=\omega_{+}-\omega_{-}+\phi-\Delta f_{2} \leq \omega+2\left[K-K_{0}\right] e^{f} e^{u},
$$

whence

$$
\sup _{U \subset E}\left\{\int_{U}(-\Delta \psi)\right\} \leq 2 \mathcal{K}_{+}\left(E ; K_{0}\right)
$$



and we can apply the generalized Huber's inequality (2.2), to conclude that,

$$
\left(\int_{\Gamma\left(\eta^{*}(s)\right)} d \sigma\right)^{2} \geq\left[4 \pi-2 \mathcal{K}_{+}\left(E ; K_{0}\right)\right] \mu\left(\eta^{*}(s)\right) \equiv\left[4 \pi-2 \mathcal{K}_{+}\left(E ; K_{0}\right)\right] s,
$$

for any $s \in I^{*} \cap(0, \mu(0))$.

Remark 3.3. If $4 \pi-2 \mathcal{K}\left(E ; K_{0}^{+}\right)<0$, then (3.22) trivially satisfied.

To simplify the exposition let us set,

$$
2 \gamma_{E}\left(K_{0}\right)=4 \pi-2 \mathcal{K}_{+}\left(E ; K_{0}\right) .
$$

Hence, substituting (3.22) in (3.19), we obtain,

$$
2 \gamma_{E}\left(K_{0}\right) s \leq\left(-\frac{d \eta^{*}(s)}{d s}\right)^{-1} F(s), \text { for any } s \in I^{*} \cap(0, \mu(0)) \text {. }
$$

So, multiplying by $\frac{d F(s)}{d s}\left(-\frac{d \eta^{*}(s)}{d s}\right)$, we come up with the inequality,

$$
2 \frac{d F(s)}{d s}\left(\frac{d \eta^{*}(s)}{d s}\right) \gamma_{E}\left(K_{0}\right) s+\frac{d F(s)}{d s} F(s) \geq 0, \text { for any } s \in I^{*} \cap(0, \mu(0)),
$$

and conclude that,

$$
\frac{d}{d s}\left[2 \gamma_{E}\left(K_{0}\right) s \frac{d F(s)}{d s}-2 \gamma_{E}\left(K_{0}\right) F(s)+\frac{1}{2}(F(s))^{2}\right] \geq 0,
$$

for any $s \in I^{*} \cap(0, \mu(0))$. Let $P_{+}(s)$ denote the functions in the square brackets. Since $F$ and $\eta^{*}$ are both continuous and locally Lipschitz continuous in $[0, \mu(0)]$ and since, in view of (3.17), $\frac{d F(s)}{d s}$ is continuous and locally Lipschitz continuous in $[0, \mu(0)]$ as well, then we come up with the inequality,

$$
P_{+}(\mu(0))-P_{+}(0) \geq 0 .
$$

Therefore we can use (3.15), (3.16) and (3.17) to obtain,

$$
\left[2 \gamma_{E}\left(K_{0}\right) \mu(0) 2 K_{0} e^{\eta^{*}(\mu(0))}-2 \gamma_{E}\left(K_{0}\right)\left(2 K_{0} M(E)\right)+2\left(K_{0}\right)^{2} M^{2}(E)\right] \geq 0 .
$$

Since $\eta^{*}(\mu(0))=0$, this is equivalent to the following inequality,

$$
2 \gamma_{E}\left(K_{0}\right) \mu(0)-2 \gamma_{E}\left(K_{0}\right) M(E)+K_{0} M^{2}(E) \geq 0 .
$$

So, by using the inequality (3.22) once more and (3.10) we find,

$$
\begin{gathered}
L^{2}(\partial E)=\left(\int_{\partial E} e^{\frac{u}{2}} d s\right)^{2} \equiv\left(\int_{\Gamma(0)} d \sigma\right)^{2} \geq 2 \gamma_{E}\left(K_{0}\right) \mu(0) \geq \\
2 \gamma_{E}\left(K_{0}\right) M(E)-K_{0} M^{2}(E)=\left(4 \pi-2 \mathcal{K}_{+}\left(E ; K_{0}\right)-K_{0} M(E)\right) M(E),
\end{gathered}
$$


which is (1.8) as claimed.

\section{Step 4.}

We will discuss here the case where the equality holds in (1.8).

First of all, there is no chance to have the equality in (1.8) if the strict inequality holds in (3.21). Therefore, because of (3.20), we see that we must have $\phi=0$ for a.a. $x \in E$, that is, in view of (3.3) and (3.4), we also conclude that $u$ must be a solution of (1.2) in $E$, and not just a subsolution as in (1.7).

Next we must have the equality sign in the Huber's inequality used in (3.22) for a.a. $s \in$ $I^{*} \cap(0, \mu(0))$ and in (3.23) for $s=\mu(0)$. Therefore, in view of (2.1) and (2.2), we conclude that for each $t \in I \cup\{0\}$, we have,

(a) $\Omega(t)$ is simply connected and $\psi(z)=c_{t}+2 \log \left|\Phi_{t}^{\prime}(z)\left(\Phi_{t}(z)\right)^{-\alpha_{\Omega(t)}}\right|, z \in \overline{\Omega(t)}$, where $\alpha_{\Omega(t)}=\frac{1}{2 \pi} \mathcal{K}_{+}\left(\Omega(t) ; K_{0}\right), c_{t} \in \mathbb{R}$ and $\Phi_{t}$ is a conformal map of $\Omega(t)$ onto the disk of unit radius $|\mathrm{w}|=\left|\Phi_{t}(z)\right|<1$ with $\Phi_{t}\left(z_{t}\right)=0$, for some $z_{t} \in \Omega(t)$. Here $\psi$ is the function defined in (3.7). Since $\phi$ vanishes, then we have the equality sign in (3.20) and (3.21) which therefore do not provide other conditions. However, in view of the Sard's Lemma for Sobolev functions, we can assume w.l.o.g. that $\Omega(t)$ is simple for each $t \in I \cup\{0\}$, so that each $\Phi_{t}$ can be extended to a univalent and continuous map from $\overline{\Omega(t)}$ to a closed unit disk, see for example Theorem 2.6 in [20]. At this point, by setting $\mathrm{w}=\Phi_{0}(z)$, and in view of $(a)$, we conclude that,

$$
v(\mathrm{w}):=\eta\left(\Phi_{0}^{-1}(\mathrm{w})\right),
$$

is a strong solution of,

$$
-\Delta v=2 K_{0} e^{c_{0}}|\mathrm{w}|^{-2 \alpha} e^{v} \text { in }\{|\mathrm{w}|<1\}, \quad v=0 \text { on }|w|=1,
$$

where $\alpha=\alpha_{E} \equiv \alpha_{\Omega(0)}$. In particular we have that the level lines of $v$ are concentric circles centred at the origin, that is, $v$ is radial. Actually, by using the Brezis-Merle estimates for Liouville type equations (see Remark 5 in [11) and standard elliptic theory, we see that $v$ is analytic far away from the origin and of class $W^{2, q}\left(B_{1}\right)$, for a suitable $q>1$ depending on $\alpha$. Thus, by a straightforward evaluation we find that,

$$
v(\mathrm{w})=\log \frac{\tau_{0}^{2}}{\left(1+\frac{K_{0} e^{c} \tau_{0}^{2}}{4(1-\alpha)^{2}}|w|^{2(1-\alpha)}\right)^{2}},|\mathrm{w}|<1,
$$

for a suitable constant $\tau_{0} \neq 0$, to be fixed in order to satisfy the Dirichlet boundary condition. As a consequence we find,

$$
\eta(z)=\log \frac{\tau^{2} e^{-c_{0}}}{\left(1+\frac{K_{0} \tau^{2}}{4(1-\alpha)^{2}}\left|\Phi_{0}(z)\right|^{2(1-\alpha)}\right)^{2}}, z \in E,
$$


for some $\tau \neq 0$ and then, since in particular $e^{\psi(z)}=e^{c_{0}}\left|\Phi_{0}^{\prime}(z)\left(\Phi_{0}(z)\right)^{-\alpha}\right|^{2}$, we see that,

$$
\eta(z)=\log \frac{\tau^{2} e^{-\psi(z)}\left|\Phi_{0}^{\prime}(z)\left(\Phi_{0}(z)\right)^{-\alpha}\right|^{2}}{\left(1+\frac{K_{0} \tau^{2}}{4(1-\alpha)^{2}}\left|\Phi_{0}(z)\right|^{2(1-\alpha)}\right)^{2}}, z \in E .
$$

Since $\eta+\psi=f+u$, then we finally conclude that

$$
e^{f(z)+u(z)}=\frac{\tau^{2}\left|\Phi_{0}^{\prime}(z)\left(\Phi_{0}(z)\right)^{-\alpha}\right|^{2}}{\left(1+\frac{K_{0} \tau^{2}}{4(1-\alpha)^{2}}\left|\Phi_{0}(z)\right|^{2(1-\alpha)}\right)^{2}}, z \in E,
$$

as claimed. Finally, by using the well known fact that the logarithm of the modulus of a non vanishing holomorphic function is harmonic, we find,

$$
\begin{gathered}
2 K e^{f+u}=-\Delta u= \\
\Delta f-\Delta \log \left(\left|\Phi_{0}^{\prime}(z)\left(\Phi_{0}(z)\right)^{-\alpha}\right|^{2}\right)+2 \Delta \log \left(1+\frac{K_{0} \tau^{2}}{4(1-\alpha)^{2}}\left|\Phi_{0}(z)\right|^{2(1-\alpha)}\right)= \\
\Delta f+4 \pi \alpha \delta_{z=z_{0}}+2 K_{0} e^{f+u}=-\omega+4 \pi \alpha \delta_{z=0}+2 K_{0} e^{f+u},
\end{gathered}
$$

in the sense of distributions in $E$ and classically in $E \backslash\{0\}$. Therefore, if $\omega \perp e^{f+u} \mathcal{H}^{2}$, then this identity can be satisfied if and only if,

$$
2 K e^{f+u} \equiv 2 K_{0} e^{f+u} \text { for a.a. } z \in E,
$$

and $\omega=4 \pi \alpha \delta_{z=z_{0}}$. In other words

$$
f(z)=h(z)+2 \alpha G\left(z, z_{0}\right)=h(z)-2 \alpha \log \left|\Phi_{0}(z)\right|,
$$

for some $h$ harmonic in $E$. At this point (3.24) and (3.25) readily imply that $K \equiv K_{0}$ for a.a. $z \in E$.

\section{The proof of Corollary 1.7.}

(i) In this situation we just know that $u \in L_{\text {loc }}^{1}(\Omega)$ and $K e^{f+u} \in L_{\text {loc }}^{1}(\Omega)$. So we also have $\Delta u \in L_{\text {loc }}^{1}(\Omega)$ and then in particular, by the Green's representation formula, $|\nabla u| \in L_{\text {loc }}^{1}(\Omega)$. By Remark 2 in [11] we find $e^{t|u|} \in L_{\text {loc }}^{1}(\Omega)$ for any $t \geq 1$ and letting $\Omega_{0} \Subset \Omega$ be any open, smooth and relatively compact subset, we have $u \in L^{1}\left(\partial \Omega_{0}\right)$ by standard trace embeddings. Let $u=u_{1}+u_{2}$, where $u_{1}$ is the unique weak solution (in the sense of Stampacchia [24]) of the Dirichlet problem,

$$
\left\{\begin{array}{l}
-\Delta u_{1}=2 K e^{f+u} \text { in } \Omega_{0} \\
u_{1}=0 \text { on } \Omega_{0}
\end{array}\right.
$$


and $u_{2}$ satisfies,

$$
\left\{\begin{array}{l}
-\Delta u_{2}=0 \text { in } \Omega_{0}, \\
u_{2}=u \text { on } \Omega_{0} .
\end{array}\right.
$$

Then $u_{2}(x)=-\int_{\partial \Omega_{0}} u(y) \frac{\partial G_{0}}{\partial \nu}(x-y) d \ell_{y}$, where $G_{0}$ is the Green's function of $-\Delta$ relative to $\Omega_{0}$, and since $u \in L^{1}\left(\partial \Omega_{0}\right)$, then $u_{2} \in L_{\text {loc }}^{\infty}\left(\Omega_{0}\right)$. Moreover, $u_{1} \in W_{0}^{1, r}\left(\Omega_{0}\right)$ for any $r \in(1,2)$ by the results in [24] and then we find $u \in W_{\text {loc }}^{1, r}(\Omega)$.

(ii) Let $E$ be any relatively compact and simple subset, we can find an open, smooth, simple and relatively compact subset $\Omega_{1}$ such that $E \Subset \Omega_{1} \Subset \Omega$. Let $K_{n} \in C^{0}\left(\overline{\Omega_{1}}\right)$ be any sequence satisfying,

$$
K_{n} \leq K \text { a.e. in } \Omega_{1} \text { and } K_{n} e^{f+u} \rightarrow K e^{f+u} \text {, as } n \rightarrow+\infty \text {, in } L^{1}\left(\Omega_{1}\right) .
$$

Next, let $v_{n}=v_{n, 1}+u_{2}$, where $v_{n, 1}$ is the unique weak solution (in the sense of Stampacchia [24]) of the Dirichlet problem,

$$
\left\{\begin{array}{l}
-\Delta v_{n, 1}=2 K_{n} e^{f+u} \quad \text { in } \Omega_{1}, \\
v_{n, 1}=0 \text { on } \Omega_{1},
\end{array}\right.
$$

and $u_{2}$ satisfies,

$$
\left\{\begin{array}{l}
-\Delta u_{2}=0 \quad \text { in } \Omega_{1}, \\
u_{2}=u \text { on } \Omega_{1} .
\end{array}\right.
$$

Obviously, as in $(i)$ we find $u_{2} \in L_{\text {loc }}^{\infty}\left(\Omega_{1}\right)$. In particular, by the Green's representation formula, it is not difficult to see that,

$$
v_{n} \leq u \text { a.e. in } \Omega_{1} .
$$

Let us observe that, by Theorem 4.4, $e^{f+u}=e^{\rho} \in L_{\text {loc }}^{p_{0} \text { loc }}\left(\Omega \backslash S_{2 \pi}\right) \cap L_{\text {loc }}^{q_{0}}(\Omega)$ for some $p_{0}>2$ and $q_{0}>1$, whence by standard elliptic estimates and the Sobolev embedding we find $v_{n} \in$ $W^{2, q_{0}}\left(\Omega_{1}\right) \cap C^{0}\left(\overline{\Omega_{1}}\right)$. By using (3.26) with well known results in [24], we conclude that $v_{n} \rightarrow u$ in $W_{\text {loc }}^{1, r}\left(\Omega_{1}\right)$, for any $r \in(1,2)$. At this point we observe that $v_{n}$ is a solution of,

$$
-\Delta v_{n}=2 \widehat{K_{n}} e^{f} e^{v_{n}} \text { in } \Omega_{1}
$$

where,

$$
\widehat{K_{n}}=K_{n} e^{u-v_{n}} \text { satisfies } \sup _{\Omega_{1}}\left|\widehat{K_{n}}\right| \leq C_{n} e^{u} .
$$

By $(i)$ we have $\widehat{K_{n}} \in L^{t}\left(\Omega_{1}\right)$ for any $t \geq 1$. On the other side, by Proposition 1 .4, we also find that $e^{f} \in L_{\text {loc }}^{s, l o c}\left(\Omega \backslash S_{2 \pi}\right) \cap L_{\text {loc }}^{m}(\Omega)$ for some $s>2$ and $m>1$. Therefore we can apply Theorem 
1.5 $(i)$ on $\Omega_{1}$ with $K=\widehat{K_{n}}$ and $u=v_{n}$, to conclude that,

$$
\left(\int_{\partial E} e^{\frac{f+v_{n}}{2}} d \ell\right)^{2} \geq\left(4 \pi-2 \mathcal{K}_{+, n}\left(E ; K_{0}\right)-K_{0} \int_{E} e^{f+v_{n}}\right) \int_{E} e^{f+v_{n}},
$$

where,

$$
\mathcal{K}_{+, n}\left(E ; K_{0}\right)=k_{s,+}(E)+\int_{E}\left[\widehat{K_{n}}-K_{0}\right]^{+} e^{f+v_{n}} d x .
$$

Since $v_{n} \rightarrow u$ in $W_{\text {loc }}^{1, r}\left(\Omega_{1}\right)$ and in view of (3.26), along a subsubsequence (which we will not relabel) we have $v_{n} \rightarrow u$ a.e. in $\Omega_{1}$ and $K_{n} e^{f+u} \rightarrow K e^{f+u}$, as $n \rightarrow+\infty$, a.e. in $\Omega_{1}$. Then, by (3.27) and the dominated convergence theorem we conclude that,

$$
\begin{aligned}
\int_{E} e^{f+v_{n}} & \rightarrow \int_{E} e^{f+u}, \text { as } n \rightarrow+\infty, \\
\mathcal{K}_{+, n}\left(E ; K_{0}\right) & \rightarrow \mathcal{K}_{+}\left(E ; K_{0}\right) \text {, as } n \rightarrow+\infty,
\end{aligned}
$$

and,

$$
\int_{\partial E} e^{\frac{f+v_{n}}{2}} d \ell \rightarrow \int_{\partial E} e^{\frac{f+u}{2}} d \ell
$$

where for the second limit we observe that,

$$
\left[\widehat{K_{n}}-K_{0}\right]^{+} e^{f+v_{n}}=\left[K_{n} e^{u-v_{n}}-K_{0}\right]^{+} e^{f+v_{n}} \leq\left[K e^{u-v_{n}}\right]^{+} e^{f+v_{n}}=[K]^{+} e^{f+u} .
$$

It is understood that the last limit holds true whenever $\int_{\partial E} e^{\frac{f+u}{2}} d \ell$ is finite, otherwise (1.8) is trivially satisfied since $M(E)<+\infty$. Therefore, in the limit $n \rightarrow+\infty$, along the given subsequence we recover (1.8), as claimed.

\section{Application to the Alexandrov's isoperimetric inequality.}

The notion of Surface of Bounded Curvature ( $S B C$ for short) was introduced by A.D. Alexandrov [2], as a model to describe surfaces with a wide variety of singularities. A detailed discussion of this subtle subject is behind the scope of our work, and we refer the reader to [3] and [22] for a complete account about the subject, and to [30] for a shorter exposition of some of the main results. Here we will just use an equivalent local description of these objects.

Indeed, according to a series of results due to Huber and Reshetnyak, see [22], an $S B C$ without boundary can be equivalently defined as a Riemann surface $\mathcal{M}$ equipped with a metric $\mathfrak{g}$, which admits an atlas of local charts $\mathcal{U}=\left\{U_{j}, \Phi_{j}\right\}_{j \in J}$, such that each $\Phi_{j}$ is an isometry of $U_{j}$ on $\Omega_{j}=\Phi_{j}\left(U_{j}\right)$, with $\Omega_{j} \subset \mathbb{R}^{2}(\simeq \mathbb{C})$, a smooth, open and bounded set, such that $\mathfrak{g}$ in local 
coordinates takes the form of a quadratic differential, $\Phi_{j}^{\#}(\mathfrak{g})=e^{\rho_{j}(z)}|d z|^{2}, z=x+i y \in \mathbb{C}$. Here \# denotes the standard pull-back, $|d z|^{2}$ is the Euclidean metric and $\rho \equiv \rho_{j}=\rho_{+}-\rho_{-}$, where $\rho_{ \pm}$are two superharmonic functions defined by,

$$
\rho_{ \pm}(z)=h_{ \pm}^{0}(z)+\int_{\Omega_{j}} \Gamma(z, y) d \omega_{ \pm}^{0}(y), \quad \Gamma(z, y)=\frac{1}{2 \pi} \log \left(\frac{1}{|z-y|}\right),
$$

with $h_{ \pm}^{0}$ harmonic in $\Omega_{j}$. Here $\omega_{ \pm}^{0}$ are the mutually orthogonal non negative measures defined by the Jordan decomposition of a measure of bounded total variation on $\Omega_{j}, \omega^{0}=\omega_{+}^{0}-\omega_{-}^{0}$. Any such system of coordinates is said to be isothermal and any metric taking the form $e^{\rho(z)}|d z|^{2}$ with $\rho$ as in (4.1) is said to be subharmonic. Among other things, the definition is completed by the transitions rules between charts of functions and holomorphic forms, thus including the metric, see [22] for further details.

This is why we will focus our attention on the local model of an $S B C$.

Definition 4.1. An Abstract Surface of Bounded Curvature (ASBC for short) is a pair $\mathcal{S}=$ $\left\{\Omega, e^{\rho(z)}|d z|^{2}\right\}$, where $\Omega \subset \mathbb{R}^{2}$ is open, smooth and bounded and $\rho=\rho_{+}-\rho_{-}$, with $\rho_{ \pm}$as defined in (4.1).

So, if $\mathcal{S}=\left\{\Omega, e^{\rho(z)}|d z|^{2}\right\}$ is an $A S B C$, according to Reshetnyak (see [22] Theorem 8.1.7), the total curvature $\mathcal{K}$, is the measure of finite total variation defined as follows,

Definition 4.2. Let $\mathcal{S}=\left\{\Omega, e^{\rho(z)}|d z|^{2}\right\}$ be an $A S B C$. The total curvature $\mathcal{K}(E)$ of a Borel set $E \subseteq \Omega$ is defined by:

$$
2 \mathcal{K}(E):=\omega^{0}(E)=\omega_{+}^{0}(E)-\omega_{-}^{0}(E)
$$

Remark 4.3. We remark that, with this definition, the total curvature is well defined and finite for any Borel set $E \subseteq \Omega$. Nevertheless, if for some $z_{0} \in \Omega$ it holds $\omega_{+}^{0}\left(z_{0}\right) \geq 4 \pi$, then the lengths and areas of sets containing $z_{0}$, as defined via the metric $g=e^{\rho(z)}|d z|^{2}$ (see (4.6), (4.7) below) are not well defined in general. Any point $z_{0} \in \Omega$ which satisfies $\omega_{+}^{0}\left(z_{0}\right) \geq 4 \pi$ is said to be a cusp.

From now on we will assume that $\mathcal{S}=\left\{\Omega, e^{\rho(z)}|d z|^{2}\right\}$ is an $A S B C$ with no cusps, that is, we assume that,

$$
\forall z \in \Omega, \omega_{+}^{0}(z)<4 \pi .
$$

Let $S_{2 \pi}=\left\{x \in \Omega: \omega_{+}^{0}(z) \geq 2 \pi\right\}$. We have the following seemingly new result about the structure of subharmonic metrics with no cusps. Interestingly enough it is sharp, see Example 1 
below for further details. The proof is based on various results and arguments in [11] about the regularity properties of Liouville-type equations. Here $\mathcal{H}^{\gamma}$, with $\gamma>0$, denotes the $\gamma$-dimensional Hausdorff measure.

Theorem 4.4. Let $\mathcal{S}=\left\{\Omega, e^{\rho(z)}|d z|^{2}\right\}$ be an $A S B C$ with no cusps.

Then $e^{\rho} \in L_{\text {loc }}^{p_{0} \text { loc }}\left(\Omega \backslash S_{2 \pi}\right) \cap L_{\text {loc }}^{q_{0}}(\Omega)$ for some $p_{0}>2$ and some $q_{0}>1$. Moreover, there exists $K \in L_{\text {loc }}^{1}\left(\Omega ; e^{\rho} \mathcal{H}^{2}\right)$ and a Radon measure $k_{s}$ on $\Omega$, satisfying $k_{s} \perp e^{\rho} \mathcal{H}^{2}$, such that, letting $k_{s}=k_{s,+}-k_{s,-}$ be the Jordan decomposition of $k_{s}$, then $\rho$ can be decomposed as $\rho=u+f$, where $f=f_{+}-f_{-}$, with $f_{ \pm}$satisfying (1.1) with $\omega_{ \pm}=2 k_{s, \pm}$ and $h_{ \pm}$suitable harmonic functions and where $u \in L_{\text {loc }}^{1}(\Omega)$ is a solution of,

$$
-\Delta u=2 K e^{f+u} \quad \text { in } \Omega
$$

in the sense of distributions. In particular, either,

(i) $K \in L_{\mathrm{loc}}^{s}(\Omega)$, for some $s>\frac{q_{0}}{q_{0}-1}$ and then $u$ is a strong solution of (4.3) which satisfies $u \in L_{\mathrm{loc}}^{\infty}(\Omega) \cap W_{\mathrm{loc}}^{2, r}(\Omega), \forall 1 \leq r \leq \frac{s q_{0}}{s+q_{0}}$, or,

(ii) $u \in W_{\mathrm{loc}}^{1, r}(\Omega)$ for any $r \in(1,2)$ and $e^{t|u|} \in L_{\mathrm{loc}}^{1}(\Omega)$ for any $t \geq 1$.

In both cases,

$$
e^{\rho(z)}|d z|^{2} \equiv e^{u(z)+f(z)}|d z|^{2}, z \in \Omega, \quad K e^{f+u} \in L_{\mathrm{loc}}^{1}(\Omega),
$$

and

$$
\mathcal{K}(E)=\int_{E} K e^{f+u}+k_{s}(E),
$$

for any relatively compact Borel set $E \Subset \Omega$. Moreover, if $\rho=u+f$ for a pair $\{u, f\}$ as above, then, for any fixed $h$ harmonic in $\Omega$, the pair $\left\{u_{h}, f_{h}\right\}:=\{u-h, f+h\}$ satisfies the same properties with $\rho=u_{h}+f_{h}$.

Proof. Let $H(z, y)=G(z \cdot y)-\Gamma(z, y)$ be the regular part of the Green's function on $\Omega$. Then $m_{ \pm}(z)=\int_{\Omega} H(z, y) d \omega_{ \pm}^{0}(y)$ are harmonic in $\Omega$, and $\rho+m_{+}-m_{-}$takes the form $\rho_{+}-\rho_{-}$for a suitable pair $\rho_{ \pm}$satisfying (1.1). Therefore, by Proposition 1.4 we find $e^{\rho} \in L_{\text {loc }}^{p_{0} \text { loc }}\left(\Omega \backslash S_{2 \pi}\right) \cap$ $L_{\text {loc }}^{q_{0}}(\Omega)$ for some $p_{0}>2$ and some $q_{0}>1$. Then $e^{\rho} \mathcal{H}^{2}$ is a Radon measure on $\Omega$, and so it is well defined the Lebesgue decomposition of $\mathcal{K}$ with respect to $e^{\rho} \mathcal{H}^{2}$,

$$
\mathcal{K}=K e^{\rho} \mathcal{H}^{2}+k_{s}, \quad K \in L_{\text {loc }}^{1}\left(\Omega ; e^{\rho} \mathcal{H}^{2}\right), \quad k_{s} \perp e^{\rho} \mathcal{H}^{2},
$$

where $k_{s}$ is a Radon measure on $\Omega$. We first observe that, since $\rho \in L_{\text {loc }}^{1}(\Omega)$, then $-\Delta \rho=\omega_{+}^{0}-\omega_{-}^{0}$ holds in the sense of distributions in $\Omega$, whence, by (4.5) and the definition of $\mathcal{K}$, we see that the following equality,

$$
-\Delta \rho=2 K e^{\rho}+2 k_{s}
$$


holds as well, in the sense of distributions in $\Omega$. Let $f=f_{+}-f_{-}$be defined by (1.1) with $\omega_{ \pm}=2 k_{s, \pm}, h_{ \pm}=0$, and let us set,

$$
u:=\rho-f .
$$

Clearly $u \in L_{\text {loc }}^{1}(\Omega)$, and since $-\Delta f=2 k_{s}$ in the sense of distributions, then we deduce that,

$$
-\Delta u=2 K e^{f+u}+2 k_{s}+\Delta f=2 K e^{f+u},
$$

that is, $u$ satisfies (4.3) in the sense of distributions in $\Omega$.

At this point, the fact that $\mathcal{K}(E)$ takes the form (4.4) is a straightforward consequence of the fact that $k_{s} \perp e^{\rho} \mathcal{H}^{2}$. Moreover we observe that, if $K$ satisfies the assumption in $(i)$, then all the assumptions of Lemma $3.1(a)$ are satisfied and then the conclusion readily follows.

So we are left with the case where $K$ does not satisfy the assumption in $(i)$, that is, we just know that $K \in L^{1}\left(\Omega ; e^{f+u} \mathcal{H}^{2}\right)$ where $u \in L^{1}(\Omega)$ is a solution of (4.3) in the sense of distributions. Therefore all the assumptions of Corollary 1.7 $(i)$ are satisfied and then the desired conclusion follows.

Finally it is obvious that the representation $\rho=u+f$ with all the properties established above still holds for $\left\{u_{h}, f_{h}\right\}$ where $h$ is an arbitrary harmonic function in $\Omega$.

Let $E \Subset \Omega$ be any regular and relatively compact subset and suppose that (4.2) holds. Then we define the length of $\partial E$,

$$
L(\partial E)=\int_{\partial E} e^{\frac{f+u}{2}} d \ell
$$

and the area of $E$,

$$
M(E)=\int_{E} e^{f+u} d x .
$$

Definition 4.5. For $K_{0} \in \mathbb{R}$ and for any and relatively compact Borel set $E \Subset \Omega$, we define the positive variation of the total curvature of $E$ with respect to $K_{0}$,

$$
\mathcal{K}_{+}\left(E ; K_{0}\right)=\sup _{U \subseteq E}\left\{\mathcal{K}(U)-K_{0} \int_{U} e^{f+u} d x\right\},
$$

where the supremum is taken over all Borel sets $U \subseteq E$.

Because of (4.4), and since $k_{s,+} \perp e^{f+u} \mathcal{H}^{2}$, then $\mathcal{K}_{+}\left(E ; K_{0}\right)$ takes the form,

$$
\mathcal{K}_{+}\left(E ; K_{0}\right)=k_{s,+}(E)+\int_{E}\left[K-K_{0}\right]^{+} e^{f+u} d x .
$$


Definition 4.6. For fixed $\alpha>-1$ and $K_{0}>0$, a spherical $\left\{K_{0}, \alpha\right\}$-cone is the ASBC defined by $\left\{B_{1},|\mathrm{w}|^{-2 \alpha} e^{v(\mathrm{w})}|d \mathrm{w}|^{2}\right\}$ where $B_{1}=\{\mathrm{w} \in \mathbb{C}:|\mathrm{w}|<1\}$ and,

$$
e^{v(\mathrm{w})}=\frac{\tau_{0}^{2}}{\left(1+\frac{K_{0} \tau_{0}^{2}}{4(1-\alpha)^{2}}|\mathrm{w}|^{2(1-\alpha)}\right)^{2}},|\mathrm{w}|<1,
$$

for some $\tau_{0} \neq 0$.

It is worth to remark that the function $v$ in Definition 4.6 is of class $\left.L^{\infty}\left(B_{1}\right) \cap W_{\text {loc }}^{2, p}\left(B_{1} \backslash\{0\}\right\}\right) \cap$ $W^{2, q}\left(B_{1}\right)$ for any $p>2$ and for any $q<\frac{1}{|\alpha|}$ and it is a strong solution of $-\Delta v=2 K_{0}|\mathrm{w}|^{-2 \alpha} e^{v}$ in $B_{1}$.

In view of Theorem 1.5, Corollary 1.7 and Theorem 4.4, and in the same spirit of [4, for $K_{0} \geq 0$ we obtain a new proof of the Alexandrov [2] isoperimetric inequality on an ASBC. At least to our knowledge the characterization of the equality sign in this weak framework is new.

Theorem 4.7. Let $\mathcal{S}=\left\{\Omega, e^{\rho}|d z|^{2}\right\}$ be an $A S B C$ with no cusps and fix $K_{0} \geq 0$. Then the curvature takes the form (4.4) for some $u, f, K, k_{s}$ as in Theorem 4.4 and for any simple and relatively compact subset $E \Subset \Omega$, it holds,

$$
L^{2}(\partial E) \geq\left(4 \pi-2 \mathcal{K}_{+}\left(E ; K_{0}\right)-K_{0} M(E)\right) M(E) .
$$

In particular, if $K$ satisfies the assumption of Theorem 4.4 $(i)$ and also $K \in L_{\text {loc }}^{n \text {,loc }}\left(\Omega \backslash S_{2 \pi}\right)$, for some $n>\frac{2 p_{0}}{p_{0}-2}$, then the equality in (4.9) holds if and only if:

- $\left\{E, e^{\rho}|d z|^{2}\right\}$ is isometric to a spherical $\left\{K_{0}, \alpha\right\}$-cone with $\alpha=\frac{1}{2 \pi} k_{s,+}(E)$;

- $\rho=u+f$ and $e^{f+u}$ takes the form (1.9), where $u$ is a solution of (4.3) with $K \equiv K_{0}$ for a.a. $z \in$ $E$ and $k_{s}=2 \pi \alpha \delta_{z=z_{0}}$, for some $z_{0} \in E$, that is, $f(z)=h(z)+2 \alpha G\left(z, z_{0}\right)=h(z)-2 \alpha \log \left|\Phi_{0}(z)\right|$, for some function $h$ harmonic in $E$.

Proof. Since $\mathcal{S}$ is an $A S B C$ with no cusps, then, by Theorem 4.4, the curvature takes the form (4.4) where $u \in L_{\mathrm{loc}}^{1}(\Omega)$ is a solution of (4.3) in the sense of distributions, $f$ takes the form (1.1) with $h_{ \pm}$harmonic and $\omega_{ \pm}=2 k_{s, \pm}$ and $K e^{u+f} \in L^{1}(\Omega)$. If $K$ satisfies the assumption in Theorem $4.4(i)$ and also $K \in L_{\text {loc }}^{n \text {,loc }}\left(\Omega \backslash S_{2 \pi}\right)$, for some $n>\frac{2 p_{0}}{p_{0}-2}$, then all the hypothesis of Theorem 1.5(i) are satisfied as well. As a consequence, the inequality (4.9) holds and the equality sign is attained if and only if (1.9) holds, that is,

$$
e^{f(z)+u(z)}|d z|^{2}=\left|\sigma \Phi_{0}(z)\right|^{-2 \alpha} e^{v\left(\sigma \Phi_{0}(z)\right)}\left|d\left(\sigma \Phi_{0}(z)\right)\right|^{2}=|\mathrm{w}|^{-2 \alpha} e^{v(\mathrm{w})}|d \mathrm{w}|^{2}, \sigma=\sqrt[1-\alpha]{\tau},
$$

for any $B_{1} \ni \mathrm{w}=\Phi_{0}(z), z \in E$, as claimed. In particular, since $\omega=2 k_{s} \perp e^{u+f} \mathcal{H}^{2}$ by construction, then Theorem 1.5(iii) can be applied as well. This observation completes the discussion of the equality case.

Clearly, to conclude the proof, it is enough to show that (4.9) holds in case (ii) of Theorem 4.4 
D.B. \& D.C.

is satisfied. However this is just the content of Corollary $1.7(i i)$ which immediately yields the desired conclusion.

\section{EXAmples.}

We recall that a point $P$ on an $S B C$ is said to be a conical singularity of order $\alpha>-1$ if in an isothermal chart $\{\Omega, z\}$ such that $z(P)=0$, the metric takes the form $e^{\rho(z)}|d z|^{2}=|z|^{2 \alpha} e^{u(z)}|d z|^{2}$, where $u \in C^{0}(\Omega) \cap C^{2}(\Omega \backslash\{0\})$.

In this section $\delta_{p}$ denotes the Dirac delta with pole at $p \in \mathbb{R}^{2}$.

\section{Example 1.}

We use Example 1 in [1] to construct an ASBC $\left\{B_{1}, e^{\rho}|d z|^{2}\right\}$ such that $\left\{u, f, K, k_{s}\right\}$ as obtained in Theorem 4.4 have the following properties:

- either $e^{\rho} \in L^{\infty}\left(B_{1}\right)$ or $e^{\rho} \in L^{q}\left(B_{1}\right)$, for any $q \geq 1$;

- $K \in L^{1}\left(e^{\rho} \mathcal{H}^{2}, B_{1}\right) \cap L^{1}\left(B_{1}\right)$ but there is no $s>1$ such that $K \in L^{s}\left(B_{1}\right)$;

- $u$ is not locally bounded;

- $u$ has all the properties claimed in Theorem $4.4(i i)$.

Let $0 \neq a<1$, and for $z \in B_{1} \backslash\{0\}$ let us set, $u(z)=-a \log \left(\log \left(\frac{e}{|z|}\right)\right)$ and,

$$
K(z)=-\frac{a}{2}|z|^{-2}\left(\log \left(\frac{e}{|z|}\right)\right)^{-(2-a)} .
$$

The superharmonic function $\rho(z)=\int_{B_{1}} G(z, y) d \omega^{0}(y)$, where $\omega^{0}(y)=2 K(y) e^{u(y)} d \mathcal{H}^{2}$, takes the form $\rho=\rho_{+}-\rho_{-}$as in (4.1) with $h_{ \pm}^{0}=0$, and $\omega_{-}^{0}=0$ and $\omega_{+}^{0}(y)=2 K(y) e^{u(y)} d \mathcal{H}^{2}$ if $a<0$, while $\omega_{-}^{0}(y)=2 K(y) e^{u(y)} d \mathcal{H}^{2}$ and $\omega_{+}^{0}=0$ if $a \in(0,1)$. Since $K e^{u} \in L^{1}\left(B_{1}\right)$, then $\omega^{0}<<e^{u} \mathcal{H}^{2}$ and so we find $\left\{u, f, K, k_{s}\right\}$ as claimed in Theorem 4.4 by setting $f=0, k_{s}=0, K \equiv K$ and $u \equiv u$. In fact we see that $u$ is a solution of,

$$
\left\{\begin{array}{l}
-\Delta u=2 K e^{u} \quad \text { in } B_{1}, \\
u=0 \quad \text { on } B_{1},
\end{array}\right.
$$

that is, in particular $u \equiv \rho$, and so we find,

$$
e^{\rho(z)}=\left(\log \left(\frac{e}{|z|}\right)\right)^{-a}, z \in B_{1} .
$$

If $a \in(0,1)$, then $e^{\rho} \in L^{\infty}\left(B_{1}\right), K \in L^{1}\left(e^{\rho} \mathcal{H}^{2}, B_{1}\right) \cap L^{1}\left(B_{1}\right)$ but $u(z) \rightarrow-\infty$ as $z \rightarrow 0$. If $a<0$, then $e^{\rho} \in L^{q}\left(B_{1}\right)$ for any $q \geq 1, K \in L^{1}\left(e^{\rho} \mathcal{H}^{2}, B_{1}\right) \cap L^{1}\left(B_{1}\right)$ but $u(z) \rightarrow+\infty$ as $z \rightarrow 0$. In both cases, there is no $s>1$ such that $K \in L^{s}\left(B_{1}\right)$, so there is no chance that $K$ satisfies the assumption of Theorem 4.4 $(i)$. On the other side, in both case it is easy to check that $u$ has all 
the properties claimed in Theorem 4.4(ii). Clearly Theorem 4.7 applies and then (4.9) holds on $\left\{B_{1}, e^{\rho}|d z|^{2}\right\}$.

\section{Example 2.}

Let $\mathbb{S}_{\alpha_{1}, \alpha_{2}}^{2}$ be the $S B C$ defined by the isothermal charts $\left\{\Omega_{i}, \varphi_{i}\right\}_{i=1,2}$ and the local metrics $\left\{g_{i}\right\}_{i=1,2}$ constructed as follows. For $r_{0} \geq 4$ and $-1<\alpha_{1} \leq \alpha_{2} \leq 0$, we define,

$$
\begin{gathered}
\Omega_{2}=\left\{z \in \mathbb{C}:|z|<r_{0}\right\}, \quad \varphi_{2}=z, \quad g_{2}=e^{\rho}|d z|^{2}, \\
\Omega_{1}=\left\{z \in \mathbb{C} \cup\{\infty\}:|z|>\frac{1}{r_{0}}\right\}, \quad \varphi_{1}=\frac{1}{z}, \quad g_{1}=\varphi_{1}^{\#}\left(g_{2}\right),
\end{gathered}
$$

where,

$$
\rho(z)= \begin{cases}\log \left(\frac{4\left(1+\alpha_{2}\right)^{2}|z|^{2 \alpha_{2}}}{\left(1+|z|^{2\left(1+\alpha_{2}\right)}\right)^{2}}\right), & |z|<1, \\ \log \left(\frac{4\left(1+\alpha_{2}\right)^{2}|z|^{2 \alpha_{1}}}{\left(1+|z|^{2\left(1+\alpha_{1}\right)}\right)^{2}}\right), & |z| \in[1,+\infty) .\end{cases}
$$

This is a compact surface without boundary, homeomorphic to the two sphere, with two conical singularities, $z=\infty$ of order $\alpha_{1}$ and $z=0$ of order $\alpha_{2}$. For $\alpha_{1}=\alpha_{2}<0$ we are reduced to the classical "american football" 28], with constant Gaussian curvature $K \equiv 1$. Instead, if $\alpha_{1}<\alpha_{2} \leq 0$, we have the glueing of two caps of american footballs with gaussian curvatures 1 and $\frac{\left(1+\alpha_{1}\right)^{2}}{\left(1+\alpha_{2}\right)^{2}}$ respectively, with different conical singularities, see [7] and [15] for more details about this singular surface.

We consider a decomposition in the $\left\{\Omega_{2}, \varphi_{2}\right\}$ chart, as claimed in Theorem 4.4, of the form $\rho(z)=f(z)+u(z)$, where,

$$
u(z)= \begin{cases}\log \left(\frac{4\left(1+\alpha_{2}\right)^{2}}{\left(1+|z|^{2\left(1+\alpha_{2}\right)}\right)^{2}}\right), & |z|<1, \\ \log \left(\frac{4\left(1+\alpha_{2}\right)^{2}|z|^{2\left(\alpha_{1}-\alpha_{2}\right)}}{\left(1+|z|^{2\left(1+\alpha_{1}\right)}\right)^{2}}\right), & |z| \in[1,+\infty),\end{cases}
$$

and

$$
f(z)=f\left(z ; \alpha_{2}\right)=2 \alpha_{2} \log |z|,|z| \in(0,+\infty)
$$


Clearly we have $u \in W_{\text {loc }}^{2, k}\left(\mathbb{R}^{2} \backslash\{0\}\right) \cap W_{\text {loc }}^{2, r}\left(\mathbb{R}^{2}\right)$, for any $k>2$ and $1<r<\frac{2}{\left|\alpha_{1}\right|}$, which is also a strong solution of $-\Delta u=2 K|z|^{2 \alpha_{2}} e^{u}$ in $\mathbb{R}^{2}$, with,

$$
K(z)= \begin{cases}1, & |z| \in[0,1) \\ \frac{\left(1+\alpha_{1}\right)^{2}}{\left(1+\alpha_{2}\right)^{2}}, & |z| \in(1,+\infty)\end{cases}
$$

So $K \in L^{\infty}\left(\mathbb{R}^{2}\right)$ and putting,

$$
k_{s, 2}=2 \pi\left|\alpha_{2}\right| \delta_{z=0}
$$

we find,

$$
\mathcal{K}(E)=\int_{E} K e^{f+u} d \mathcal{H}^{2}+k_{s, 2}(E), \quad E \Subset\left\{|z|<r_{0}\right\},
$$

which is the total curvature of a relatively compact Borel set $E$ in the $\left\{\Omega_{2}, \varphi_{2}\right\}$ chart. For a generic Borel set $E_{0} \subseteq \mathbb{C} \cup\{\infty\}$, we can consider the analogue decomposition for $g_{1}$ which takes the form $g_{1}=e^{\rho_{1}}|d \mathrm{w}|^{2}$, with $\rho_{1}=f_{1}+u_{1}$, where

$$
u_{1}(\mathrm{w})= \begin{cases}\log \left(\frac{4\left(1+\alpha_{2}\right)^{2}}{\left(1+|\mathrm{w}|^{2\left(1+\alpha_{1}\right)}\right)^{2}}\right), & |\mathrm{w}|<1, \\ \log \left(\frac{4\left(1+\alpha_{2}\right)^{2}|\mathrm{w}|^{2\left(\alpha_{2}-\alpha_{1}\right)}}{\left(1+|\mathrm{w}|^{2\left(1+\alpha_{2}\right)}\right)^{2}}\right), & |\mathrm{w}| \in[1,+\infty),\end{cases}
$$

$f_{1}(\cdot)=f\left(\cdot, \alpha_{1}\right)$, and eventually find the total curvature of any Borel set $E_{0} \subseteq \mathbb{C} \cup\{\infty\}$,

$$
\mathcal{K}\left(E_{0}\right)=\int_{E_{0,2}} K e^{f+u} d \mathcal{H}^{2}+k_{s, 2}\left(E_{0,2}\right)+\int_{\varphi_{1}\left(E_{0,1}\right)} K_{1} e^{f_{1}+u_{1}} d \mathcal{H}^{2}+k_{s, 1}\left(\varphi_{1}\left(E_{0,1}\right)\right),
$$

where $E_{0,2}=E_{0} \cap\left\{|z|<r_{0}\right\}, E_{0,1}=E_{0} \cap\left\{|z| \geq r_{0}\right\}, K_{1}=K \circ \varphi_{1}$, and,

$$
k_{s, 1}=2 \pi\left|\alpha_{1}\right| \delta_{\mathrm{w}=0}
$$

Next, to simplify the notations let us set,

$$
\sigma_{1,2}=\frac{\left(1+\alpha_{1}\right)^{2}}{\left(1+\alpha_{2}\right)^{2}} \leq 1
$$

It is easy to check that the area of $\mathbb{S}_{\alpha_{1}, \alpha_{2}}^{2}$ is $2 \pi\left(1+\alpha_{2}\right)+\frac{1}{\sigma_{1,2}} 2 \pi\left(1+\alpha_{1}\right)$ while, by using (5.2), we see that the total curvature of $\mathbb{S}_{\alpha_{1}, \alpha_{2}}^{2}$ is $4 \pi$, in agreement with the fact that, as well known [3], the Gauss-Bonnet formula holds even in this singular context. Please observe that this is just an equivalent formulation of the singular Gauss-Bonnet formula, see [29], which asserts that the 
global integral of the absolutely continuous part of the Gaussian curvature equals the singular Euler characteristic, yielding in this particular case the well known identity,

$$
\int_{B_{1}} K e^{f+u} d \mathcal{H}^{2}+\int_{\varphi_{1}\left(\left(B_{1}\right)^{c}\right)} K_{1} e^{f_{1}+u_{1}} d \mathcal{H}^{2}=2 \pi\left(2+\alpha_{1}+\alpha_{2}\right) .
$$

If $E$ is a simple set surrounding the origin, then we can always take $r_{0}$ large enough to guarantee that $E \Subset\left\{|z|<r_{0}\right\}$ so that the inequality (4.9) takes the form,

$L^{2}(\partial E) \geq\left(4 \pi\left(1+\alpha_{2}\right)-2\left[1-K_{0}\right]^{+} M\left(E \cap B_{1}\right)-2\left[\sigma_{1,2}-K_{0}\right]^{+} M\left(E \cap\left(B_{1}\right)^{c}\right)-K_{0} M(E)\right) M(E)$.

In particular, if $K$ is not constant in $E$, then the inequality is always strict and if we choose $K_{0}=1$, then it reduces to the well known Bol's [10] inequality,

$$
L^{2}(\partial E) \geq\left(4 \pi\left(1+\alpha_{2}\right)-M(E)\right) M(E)
$$

If $E=B_{R}$ with $R \leq 1$, then $K \equiv 1$ in $E$ and since,

$$
L^{2}\left(\partial B_{R}\right)=\left(\int_{0}^{2 \pi} \frac{2\left(1+\alpha_{2}\right) R^{\alpha_{2}}}{1+R^{2\left(1+\alpha_{2}\right)}} d \ell\right)^{2}=\frac{16 \pi^{2}\left(1+\alpha_{2}\right)^{2} R^{2 \alpha_{2}}}{\left(1+R^{2\left(1+\alpha_{2}\right)}\right)^{2}},
$$

and,

$$
M\left(B_{R}\right)=\int_{B_{R}} \frac{4\left(1+\alpha_{2}\right)^{2}|x|^{2 \alpha_{2}}}{\left(1+|x|^{2\left(1+\alpha_{2}\right)}\right)^{2}} d x=\frac{4 \pi\left(1+\alpha_{2}\right) R^{2 \alpha_{2}}}{1+R^{2\left(1+\alpha_{2}\right)}},
$$

then we find the equality in (4.9) with $K_{0}=1$,

$$
\begin{gathered}
L^{2}\left(\partial B_{R}\right)=\frac{16 \pi^{2}\left(1+\alpha_{2}\right)^{2} R^{2 \alpha_{2}}}{\left(1+R^{2\left(1+\alpha_{2}\right)}\right)^{2}}= \\
\left(4 \pi\left(1+\alpha_{2}\right)-\frac{4 \pi\left(1+\alpha_{2}\right) R^{2 \alpha_{2}}}{1+R^{2\left(1+\alpha_{2}\right)}}\right) \frac{4 \pi\left(1+\alpha_{2}\right) R^{2 \alpha_{2}}}{1+R^{2\left(1+\alpha_{2}\right)}}=\left(4 \pi\left(1+\alpha_{2}\right)-M\left(B_{R}\right)\right) M\left(B_{R}\right) .
\end{gathered}
$$

\section{Example 3.}

This example illustrates the failure of Theorem 4.4 on a surface homeomorphic to the two-sphere with a cusp and in the same time the kind of singularity which yields a curvature function $K$ which is unbounded but in $L^{r}(E)$ for some $r>1$. 
Let us consider the same charts $\left\{\Omega_{i}, \varphi_{i}\right\}_{i=1,2}$ as in Example 2, where this time the metric $g_{2}(z)=e^{\rho(z)}|d z|^{2}$ is defined as follows,

$$
\rho(z)= \begin{cases}\log \left(\frac{2}{\left(2-|z|^{\frac{1}{2}}\right)^{2}}\right), & |z|<1, \\ \log \left(\frac{8|z|^{\frac{3}{2}}}{\left(1+|z|^{\frac{1}{2}}\right)^{2}}\right), & |z| \in[1,+\infty) .\end{cases}
$$

We consider a decomposition as claimed in Theorem 4.4 in the $\left\{\Omega_{2}, \varphi_{2}\right\}$ chart, $\rho(z)=f(z)+u(z)$, where we choose $f=0$ so that $u=\rho$, which satisfies $u \in W_{\text {loc }}^{2, k \text { loc }}\left(\mathbb{R}^{2} \backslash\{0\}\right) \cap W_{\text {loc }}^{2, r}\left(\mathbb{R}^{2}\right)$, for any $k>2$ and $1<r<\frac{4}{3}$, and is a strong solution of $-\Delta u=2 K e^{f+u}$ in $\mathbb{R}^{2}$, where,

$$
K(z)= \begin{cases}-\frac{1}{4} \frac{1}{|z|^{\frac{3}{2}}}, & |z| \in[0,1), \\ \frac{1}{32} \frac{1}{|z|^{3}}, & |z| \in(1,+\infty) .\end{cases}
$$

The total curvature of a relatively compact Borel set $E$ in the $\left\{\Omega_{2}, \varphi_{2}\right\}$ chart takes the form,

$$
\mathcal{K}(E)=\int_{E} K e^{u} d x, \quad E \Subset\left\{|z|<r_{0}\right\},
$$

with $K \in L^{r}(E) \cap L_{\text {loc }}^{\infty}(E \backslash\{0\})$ for any $1<r<\frac{4}{3}$.

On the other side, let us check whether or not the assumption (4.2) is satisfied on a generic relatively compact Borel set in the $\left\{\Omega_{1}, \varphi_{1}\right\}$ chart. The metric takes the form,

$$
g_{1}(\mathrm{w})=\varphi_{1}^{\#}\left(g_{2}\right)=e^{\rho_{1}(\mathrm{w})}|d \mathrm{w}|^{2},
$$

where,

$$
\rho_{1}(\mathrm{w})= \begin{cases}\log \left(\frac{8|\mathrm{w}|^{-\frac{9}{2}}}{\left(1+|\mathrm{w}|^{\frac{1}{2}}\right)^{2}}\right), & |\mathrm{w}| \in[0,1], \\ \log \left(\frac{2|\mathrm{w}|^{-3}}{\left(2|\mathrm{w}|^{\frac{1}{2}}-1\right)^{2}}\right), & |\mathrm{w}| \in(1,+\infty) .\end{cases}
$$

Therefore, it is readily seen that $\rho_{1}$ takes the form (4.1) with $\omega_{+}^{0}(0)=\frac{9 \pi}{2}>4 \pi$ which violates (4.2). This singular surface is still homeomorphic to the two sphere, but it has a cusp at $z=\infty$. As a consequence, while the curvature is always well defined in the sense of measures, the area of a compact Borel set in the $\left\{\Omega_{1}, \varphi_{1}\right\}$ chart is not, since $e^{\rho_{1}}$ is not an $L_{\text {loc }}^{1}\left(\mathbb{R}^{2}\right)$ function. In 
particular, there is no chance to use the argument in the proof of Theorem 4.4, which should be based on the Lebesgue decomposition of $\mathcal{K}=\omega^{0}$ with respect to $e^{\rho_{1}} \mathcal{H}^{2}$, since the latter is not even a Radon measure in this case. It is worth to mention that, nevertheless, the product $\left(K \circ \varphi_{1}\right) e^{\rho_{1}}$ is an $L_{\text {loc }}^{1}\left(\mathbb{R}^{2}\right)$ function which could be used in principle as the density of the total curvature. On the other hand, the right hand side of the Alexandrov's isoperimetric inequality (4.9) is not well defined in general.

However Theorem 4.4 and Theorem 4.7 can be applied in the $\left\{\Omega_{2}, \varphi_{2}\right\}$ chart, so that (4.9) holds therein. In particular, if $E$ is any open and relatively compact Borel set in $\Omega_{2}$, then the equality is always strict, since $K$ is never constant in $E$.

\section{REFERENCES}

[1] L. Ambrosio, J. Bertrand, On the regularity of Alexandrov surfaces with curvature bounded below. Preprint (2014).

[2] A. D. Alexandrov, Die innere Geometrie der konvexen Flächen, Springer Verlag, Berlin, 1955.

[3] A. D. Alexandrov, V.A. Zalgaller, Intrinsic Geometry of Surfaces, AMS Transl. Math. Monographs, Vol. 15, Providence, RI, 1967.

[4] C. Bandle, On a differential Inequality and its applications to Geometry, Math. Zeit. 147, (1976) 253-261.

[5] C. Bandle, Isoperimetric Inequalities and Applications, Pitman, Boston, 1980.

[6] J.L. Barbosa, M. do Carmo, A Proof of a General Isoperimetric Inequality for Surfaces, Math. Z. 162 (1978) 245-261.

[7] D. Bartolucci, On the best pinching constant of conformal metrics on $\mathbb{S}^{2}$ with one and two conical singularities, Jour. Geom. Analysis 23 (2013) 855-877.

[8] D. Bartolucci, D. Castorina, Self gravitating cosmic strings and the Alexandrov's inequality for Liouville-type equations, Comm. Cont. Math. 18(4) (2016), 1550068 (26p.).

[9] D. Bartolucci, C.S. Lin, Existence and uniqueness for Mean Field Equations on multiply connected domains at the critical parameter, Math. Ann., 359 (2014), 1-44.

[10] G. Bol, Isoperimetrische Ungleichungen für Bereiche auf Flächen, Jber. Deutsch. Math. Verein. 51, (1941), 219-257.

[11] H. Brezis \& F. Merle, Uniform estimates and blow-up behaviour for solutions of $-\Delta u=V(x) e^{u}$ in two dimensions, Comm. in P.D.E., 16(8,9) (1991), 1223-1253.

[12] J.E. Brothers \& W.P. Ziemer, Minimal rearrangement of Sobolev functions, J. Reine Angew. Math. 384 (1988), 153-179.

[13] Y.D. Burago, V.A. Zalgaller, "Geometric inequalities", Springer Ser. Sov. Math., Springer-Verlag Berlin Heidelberg 1988.

[14] De Pascale L. The Morse-Sard theorem in Sobolev spaces, Indiana Univ. Math. J., 50 (2001), 1371-1386.

[15] H. Fang, M. Lai, On curvature pinching of conic 2-spheres, Preprint (2015).

[16] D. Gilbarg, N.S. Trudinger, Elliptic Partial Differential Equations of Second Order, II Ed., Springer 2001.

[17] A. Huber, On the isoperimetric inequality on surfaces of variable Gaussian curvature, Ann. Math., 60(2) (1954), 237-247. 
D.B. \& D.C.

[18] A. Huber, Zur Isoperimetrischen Ungleichung Auf Gekrümmten Flächen, Acta. Math., 97 (1957), 95-101.

[19] R. Ossermann, Bonnesen-style Isoperimetric Inequalities, Am. Math. Mont. 86(1) (1979), 1-29.

[20] Ch. Pommerenke, Boundary Behaviour of Conformal Maps, Grandlehren der Math. Wissenschaften, 299, p. 300, Springer-Verlag, Berlin-Heidelberg, 1992

[21] Y.G. Reshetnyak, Isothermal coordinates in manifolds of bounded curvature II, Sib. Math. J., vol. 1, (1960), p. 248-276. (Russian)

[22] Y.G. Reshetnyak, Two-dimensional manifolds of bounded curvature, pp. 3-163 In: Y.G. Reshetnyak (Ed.), Geometry IV, Encyclopaedia of Math. Sci., Vol. 70, Springer (1993).

[23] T. Suzuki, Global analysis for a two-dimensional elliptic eiqenvalue problem with the exponential nonlinearly, Ann. Inst. H. Poincaré Anal. Non Linéaire 9(4) (1992), 367-398.

[24] G. Stampacchia, Le problème de Dirichlet pour les équations elliptiques du second ordre à coefficients discontinus, Ann. Ins. Fourier 15(1) (1965), pp. 189-257.

[25] G. Talenti Best Constant in Sobolev Inequality, Ann. Mat. Pura Appl., 110 (1976), pp. 353-372.

[26] P. Topping Mean curvature flow and geometric inequalities, J. Reine Angew. Math., 503, (1998) 47-61.

[27] P. Topping The isoperimetric inequality on a surface, Man. Math., 100, (1999) 23-33.

[28] M. Troyanov, Metrics of constant curvature on a sphere with two conical singularities, Proc. Third Int. Symp. on Diff. Geom. (Peniscola 1988), Lect. Notes in Math. 1410 Springer-Verlag, 296-308.

[29] M. Troyanov, Prescribing curvature on compact surfaces with conical singularities, Trans. Amer. Math. Soc. 324 (1991), 793-821.

[30] M. Troyanov, Les surfaces a courbure integrale bornee au sense d' Alexandrov, preprint arXiv:0906.3407 1.

[31] M. Troyanov, Une principe de concentration-compacité pour le suites de surfaces Riemanniens, Ann. Inst. H. Poincaré Anal. Non Lineaire 5 (1991), 419-441. 